\title{
Discrete $R$-symmetries and anomaly universality in heterotic orbifolds
}

\author{
Nana G. Cabo Bizet, ${ }^{a}$ Tatsuo Kobayashi, ${ }^{b}$ Damián K. Mayorga Peña, ${ }^{c}$ \\ Susha L. Parameswaran, ${ }^{d}$ Matthias Schmitz ${ }^{c}$ and Ivonne Zavala ${ }^{e}$ \\ ${ }^{a}$ Centro de Aplicaciones Tecnológicas y Desarrollo Nuclear, \\ Calle 30, esq.a 5ta Ave, Miramar, 6122 La Habana, Cuba \\ ${ }^{b}$ Department of Physics, Kyoto University, \\ Kyoto 606-8502, Japan \\ ${ }^{c}$ Bethe Center for Theoretical Physics and Physikalisches Institut der Universität Bonn, \\ Nussallee 12, 53115 Bonn, Germany \\ ${ }^{d}$ Department of Mathematics and Physics, Leibniz Universität Hannover, \\ Welfengarten 1, 30167 Hannover, Germany \\ ${ }^{e}$ Centre for Theoretical Physics, University of Groningen, \\ Nijenborgh 4, 9747 AG Groningen, The Netherlands \\ E-mail: nana@ceaden.edu.cu, kobayash@gauge.scphys.kyoto-u.ac.jp, \\ damian@th.physik.uni-bonn.de, \\ susha.parameswaran@itp.uni-hannover.de, \\ mschmitz@th.physik.uni-bonn.de, e.i.zavala@rug.nl
}

ABSTRACT: We study discrete $R$-symmetries, which appear in the $4 \mathrm{D}$ low energy effective field theory derived from heterotic orbifold models. We derive the $R$-symmetries directly from the geometrical symmetries of the orbifolds. In particular, we obtain the corresponding $R$-charges by requiring that the couplings be invariant under these symmetries. This allows for a more general treatment than the explicit computations of correlation functions made previously by the authors, including models with discrete Wilson lines, and orbifold symmetries beyond plane-by-plane rotational invariance. The $R$-charges obtained in this manner differ from those derived in earlier explicit computations. We study the anomalies associated with these $R$-symmetries, and comment on the results.

Keywords: Superstrings and Heterotic Strings, Discrete and Finite Symmetries, Conformal Field Models in String Theory, Anomalies in Field and String Theories

ARXIV EPRINT: 1308.5669 


\section{Contents}

1 Introduction 1

2 String orbifold CFT 2

3 Discrete $R$-symmetries from orbifold isometries 5

4 Universal $R$-symmetry anomalies $\quad 8$

5 Further $R$-symmetry candidates $\quad 11$

6 An explicit example: $\mathbb{Z}_{4}$ on $\mathrm{SO}(4)^{2} \times \mathrm{SU}(2)^{2} \quad 13$

$\begin{array}{lll}7 & \text { Discussion } & 15\end{array}$

$\begin{array}{lll}\text { A Space group elements } h_{g} \text { for the } \mathbb{Z}_{4} \text { and } Z_{6 I I} \text { orbifolds } & 17\end{array}$

\section{Introduction}

Discrete symmetries are often imposed in the context of particle physics model building beyond the Standard Model in order to forbid unwanted terms in the Lagrangian. For example, they have been invoked in order to guarantee the absence of certain operators leading to exceedingly fast proton decay. They have also been very useful for flavor physics to generate textures of quark and lepton masses and mixings. From a stringy perspective, discrete symmetries are expected to appear, either as discrete remnants of a broken gauge symmetry [1-3] or to be an inherent property of the compactification from ten to four dimensions.

In this respect, heterotic orbifold compactifications $[4,5]$ provide a phenomenologically promising UV-complete framework [6-18] that is rich in discrete symmetries, which moreover have intuitive geometric interpretations. One of the features which makes them appealing for phenomenology is the presence of $R$-symmetries. These $R$-symmetries can be understood as elements of the Lorentz group in the compact space since they treat $4 \mathrm{D}$ bosons and fermions in a different manner. Therefore they are expected not to commute with the generator of the $4 \mathrm{D} \mathcal{N}=1$ SUSY algebra. For the specific case of orbifold compactifications, one expects those rotations in $\mathrm{SO}(6)$ which are symmetries of the orbifold to manifest as $R$-symmetries of the low energy effective field theory (LEEFT).

The identification of $R$-symmetries in heterotic models appeared first in [19] for the $\mathbb{Z}_{3}$ orbifold, where they were associated to orbifold isometries which in this case were the twists acting on a single plane. Later on, more general expressions were found in [6]. However, it was recently pointed out by the authors [20], that in general, the $R$-charges 
defined in [6], receive a non-trivial contribution from the so-called gamma-phases. In the same way as gauge invariance and other selection rules have been derived $[4,5,21,22]$, the analysis in [20] was made via the explicit calculation of vanishing correlation functions, in the absence of discrete Wilson lines. A classification of the symmetries observed in orbifold geometries was presented in the appendix of [20]. There it was observed that such orbifold symmetries are exhibited by the classical instanton solutions. Thus one expects them to induce $R$-symmetries in the low energy. In the present note we determine the form of the expected $R$-charges of the physical states, by assuming that the orbifold isometries are also manifest symmetries of the LEEFT. This allows us to include discrete Wilson lines in the analysis, as well as to consider more general cases. The $R$-charges obtained in this way turn out to differ in the sign of the gamma-phase contribution, with respect to the former derivation. We provide some possible interpretations of these two results.

Anomalies of discrete symmetries have important implications in 4D field theory [2325], in particular string-derived LEEFTs. Such anomalies are expected to cancel via the Green Schwarz mechanism [26]. In heterotic orbifold models, there is only one axion available and hence, all anomalies must be universal, i.e. to cancel up to a common axion shift. ${ }^{1}$ In $[28,29]$, anomalies were studied for the discrete $R$-charges corresponding to [6]. Here, we study explicitly the anomaly conditions for the two types of $R$-charges: the one derived in this paper and the one obtained in [20]. We determine their universality for several orbifolds, factorizable and non-factorizable. While anomaly universality could be expected for the $R$-symmetries, there exists no proof that this should be indeed the case. We discuss this issue and its implications.

The paper is organized as follows: in section 2 we review briefly the orbifold CFT; in section 3 we present a derivation of $R$-symmetries from orbifold isometries, which can be applied to the more general cases in which discrete Wilson lines are present. In section 4 we compute the anomalies for the $R$-symmetries in several explicit factorizable and nonfactorizable examples. In section 5 we study further possible $R$-symmetries which appear in the low energy effective field theory due to orbifold symmetries that do not leave the fixed points invariant. We discuss these symmetries, as well as the more familiar $R$-symmetries of section 3 , by using an explicit model in 6 . In section 7 we conclude with the discussion of our results.

Note added: while this paper was in preparation, [30] appeared on the arXiv. There the $R$-symmetries of the $\mathbb{Z}_{6 I I}$ orbifold are derived and the contribution of the discrete Wilson lines is considered for the first time. The $R$-charges derived there also differ in the sign of the gamma phase contribution compared to our previous result [20]. The results presented here were obtained independently and hence confirm (and extend) those in [30].

\section{String orbifold CFT}

We begin by briefly describing the relevant elements of the string orbifold conformal field theory. Our focus will be on symmetric, six-dimensional orbifolds constructed by modding

\footnotetext{
${ }^{1}$ See [27] and references therein for such universality conditions for U(1) gauge anomalies.
} 
out a non-freely acting, Abelian isometry of the torus

$$
\mathbb{D}^{6}=\frac{\mathbb{T}^{6}}{P}=\frac{\mathbb{C}^{3} / \Lambda}{P}=\frac{\mathbb{C}^{3}}{S},
$$

where $P$ is the point group and $S=P \ltimes \Lambda$ is the space group. In order to retain $\mathcal{N}=1$ supersymmetry in four dimensions, the point group must be $\mathbb{Z}_{N}$ or $\mathbb{Z}_{N} \times \mathbb{Z}_{M}$. Here we discuss orbifolds in the first class, but our results can easily be generalized to models of the second type. For $\mathbb{Z}_{N}$ orbifolds, the generator of the point group $\theta$ can be brought to the diagonal form

$$
\theta=\operatorname{diag}\left(e^{2 \pi \mathrm{i} v^{1}}, e^{2 \pi \mathrm{i} v^{2}}, e^{2 \pi \mathrm{i} v^{3}}\right),
$$

where the coordinates of the internal space have been taken in a complexified basis $X^{i}, \bar{X}^{i}$ $(i=1,2,3)$. The twist vector $v=\left(v^{1}, v^{2}, v^{3}\right)$ is constrained by $\mathcal{N}=1$ supersymmetry to satisfy $v^{1}+v^{2}+v^{3}=0 \bmod 1$.

In the orbifold background, strings can close up to twist and lattice identifications. The closed string boundary conditions involving only lattice identifications give rise to the untwisted sector, where the relevant conformal primary fields are the identity operator; the left-moving oscillator fields $\partial X^{i}, \partial \bar{X}^{i}$ (plus their complex conjugates); the sixteen extra left-movers contributing the Cartans $\partial X^{I}(I=1, \ldots, 16)$ and the roots $e^{\mathrm{i} p \cdot X}$ of the $E_{8} \times E_{8}$ gauge symmetry; and the exponential $e^{\mathrm{i} q^{(a)} \cdot H}$, with $H$ a five-dimensional vector of free fields corresponding to the bosonized right-moving fermions and $q^{(a)}$ is either a bosonic $(a=1)$ of a fermionic $(a=1 / 2)$ weight of $\mathrm{SO}(10)$, known as $H$-momentum.

The boundary conditions of the internal space bosonic coordinates for twisted strings are of the form

$$
X^{i}\left(e^{2 \pi \mathrm{i}} z, e^{-2 \pi \mathrm{i}} \bar{z}\right)=(g X)^{i}(z, \bar{z})=\left(\theta^{k} X\right)^{i}(z, \bar{z})+\lambda^{i},
$$

for any constructing element $g=\left(\theta^{k}, \lambda\right) \in S$, where $z, \bar{z}$ are the complexified worldsheet coordinates and $\lambda^{i} \in \Lambda$. These boundary conditions correspond to strings in the $k$-th twisted sector. Notice that strings closed by $g$ and $\left\{h g h^{-1} \mid h \in S\right\}$ are physically equivalent, that is, physical twisted states are associated with conjugacy classes and not space group elements.

The standard way to deal with the branch singularities in (2.2) is to introduce twist fields $\sigma(z, \bar{z})[21,22]$ which serve to implement the local monodromy conditions,

$$
\begin{aligned}
& \partial X^{i}(z, \bar{z}) \sigma(w, \bar{w})=(z-w)^{-\bar{k}^{i}} \tau+\ldots, \\
& \partial \bar{X}^{i}(z, \bar{z}) \sigma(w, \bar{w})=(z-w)^{-k^{i}} \tilde{\tau}+\ldots,
\end{aligned}
$$

where $k^{i}=k v^{i} \bmod 1$ and $\bar{k}^{i}=\left(1-k^{i}\right) \bmod 1$, such that $0 \leq k^{i}, \bar{k}^{i}<1$, and $\tau, \tilde{\tau}$ are excited twist fields. The conformal dimensions of $\sigma$ are given by

$$
\Delta_{\sigma}=\bar{\Delta}_{\sigma}=\frac{1}{2} \sum_{i=1}^{3} k^{i}\left(1-k^{i}\right) .
$$

The twist fields for worldsheet fermions can be written in terms of the bosonized fermions as $e^{\mathrm{i} k v \cdot H}$. This leads to the definition of a shifted H-momentum:

$$
q_{s h}^{(a)}=q^{(a)}+k \cdot\left(0,0, v^{1}, v^{2}, v^{3}\right),
$$


so that the primaries in the vertex operators take the familiar form $e^{\mathrm{i} q_{s h}^{(a)} \cdot H}$. Furthermore, modular invariance requires the twist to be embedded in the gauge degrees of freedom. In fact, not only the twist but the full space group can be embedded as a shift:

$$
g=\left(\theta^{k}, n_{\alpha} e_{\alpha}\right) \mapsto V_{g}=k V+n_{\alpha} W_{\alpha},
$$

where $\left\{e_{\alpha}\right\}, \alpha=1, \ldots, 6$ spans $\Lambda, V$ is the embedding of $\theta$ and the discrete Wilson lines $W_{\alpha}$ are related to the lattice shifts [31-33]. Note that both the group laws of $S$ as well as modular invariance impose several non-trivial constraints on the choice for the embedding vectors, which are summarized for example in [34]. The relevant primary for the twisted vertices is $e^{2 \pi \mathrm{i} p_{s h} \cdot X}$, with $p_{s h}=p+V_{g}$.

To summarize, the vertex operators describing the emission of twisted states are given by:

$$
V_{-a}=e^{-a \phi}\left(\prod_{i=1}^{3}\left(\partial X^{i}\right)^{\mathcal{N}_{L}^{i}}\left(\partial \bar{X}^{i}\right)^{\overline{\mathcal{N}}_{L}^{i}}\right) e^{\mathrm{i} q_{s h}^{(a)} \cdot H} e^{\mathrm{i} p_{s h} \cdot X} \sigma,
$$

where $\phi$ is the superconformal ghost and the integers $\mathcal{N}_{L}^{i}$ and $\overline{\mathcal{N}}_{L}^{i}$ count, respectively, the number of left-moving holomorphic and anti-holomorphic oscillators present in the state. Untwisted vertex operators have the same form as those presented before but with all momenta unshifted and with the twist field replaced by the identity operator. Note that in writing (2.7), we have taken the four-dimensional momentum to zero, and neglected cocycle [35] and normalization factors, which are unimportant for our purposes.

Furthermore, invariance of the vertex operators under the full space group needs to be satisfied. Considering a certain space group element $h$, the bosonic fields transform according to

$$
\partial X^{i} \stackrel{h}{\rightarrow} e^{2 \pi \mathrm{i} v_{h}^{i}} \partial X^{i}, \quad X^{I} \stackrel{h}{\rightarrow} X^{I}+V_{h}^{I}, \quad H^{i} \rightarrow H^{i}-v_{h}^{i} .
$$

In order to see how $h$ acts on the twist fields it is convenient to decompose them into a sum of auxiliary twists $\sigma_{g}$, one for each element in the conjugacy class $[g]$

$$
\sigma \sim \sum_{g^{\prime} \in[g]} e^{2 \pi \mathrm{i} \tilde{\gamma}\left(g^{\prime}\right)} \sigma_{g^{\prime}}
$$

where the phases $\tilde{\gamma}\left(g^{\prime}\right)$ will be determined presently. For the auxiliary twists one has the following transformation behavior

$$
\sigma_{g} \stackrel{h}{\rightarrow} e^{2 \pi \mathrm{i} \Phi(g, h)} \sigma_{h g h^{-1}},
$$

with the vacuum phase $\Phi(g, h)=-\frac{1}{2}\left(V_{g} \cdot V_{h}-v_{g} \cdot v_{h}\right)$ [34]. This leads to

$$
\sigma \stackrel{h}{\rightarrow} e^{2 \pi \mathrm{i}\left[\gamma_{h}+\Phi(g, h)\right]} \sigma
$$

where we have defined

$$
\gamma_{h}=\left[\tilde{\gamma}\left(g^{\prime}\right)-\tilde{\gamma}\left(h g^{\prime} h^{-1}\right)\right] \bmod 1, \text { for some } g^{\prime} \in[g]
$$


together with the condition

$$
-\tilde{\gamma}\left(h g^{\prime} h^{-1}\right)+\tilde{\gamma}\left(g^{\prime}\right)=-\tilde{\gamma}\left(h g h^{-1}\right)+\tilde{\gamma}(g) \bmod 1,
$$

for any pair of elements $g^{\prime}, g \in[g]$. Now we can finally see how the vertex (2.7) transforms under $h$,

$$
V_{-a} \stackrel{h}{\rightarrow} \exp \left\{2 \pi \mathrm{i}\left[p_{s h} \cdot V_{h}-v_{h}^{i}\left(q_{s h}^{(a) i}-\mathcal{N}_{L}^{i}+\overline{\mathcal{N}}_{L}^{i}\right)+\gamma_{h}+\Phi(g, h)\right]\right\} V_{-a} .
$$

From (2.12) one can see that if there exists an element $g \in[g]$ which commutes with a certain $h$, then $\gamma_{h}=0 \bmod 1$, such that eq. (2.14) becomes a projection condition

$$
p_{s h} \cdot V_{h}-v_{h}^{i}\left(q_{s h}^{(a) i}-\mathcal{N}_{L}^{i}+\overline{\mathcal{N}}_{L}^{i}\right)+\Phi(g, h)=0 \bmod 1,
$$

which are the so-called orbifold GSO projectors responsible for an $\mathcal{N}=1$ supersymmetric spectrum. In all other cases, the gamma-phases can be found by demanding the transformation of $V_{-a}$ to be trivial $[33,36,37]$, i.e.

$$
\gamma_{h}=-p_{s h} \cdot V_{h}+v_{h}^{i}\left(q_{s h}^{(a) i}-\mathcal{N}_{L}^{i}+\overline{\mathcal{N}}_{L}^{i}\right)-\Phi(g, h)=\tilde{\gamma}(g)-\tilde{\gamma}\left(h g h^{-1}\right) \bmod 1 .
$$

In this way, space group invariance fixes all $\tilde{\gamma}\left(g^{\prime}\right)$ except for one, which can be reabsorbed as an overall phase in $\sigma$. Notice that there is generically more than one physical twist field $\sigma$ for each conjugacy class, given by the different linear combinations of auxiliary twist fields, in which the different gamma-phase coefficients are determined in terms of the other quantum numbers of the physical state.

\section{Discrete $R$-symmetries from orbifold isometries}

In this section we identify discrete $R$-symmetries which are expected to appear in the low energy effective field theory of a given orbifold compactification, due to symmetries in the orbifold geometry. Examples of such discrete $R$-symmetries were explicitly verified in [20], by computing correlation functions. Our present approach will instead be to assume that symmetries in the orbifold geometry give rise to $R$-symmetries in the effective field theory and - given this assumption - infer the corresponding charge conservation laws. This allows us to be more general, including symmetries beyond the plane-by-plane independent twist symmetries, as well as models with Wilson lines.

The absence of a coupling between $\mathrm{L}$ chiral superfields $\Phi_{\alpha}(\alpha=1, \ldots, \mathrm{L})$ in the superpotential of the LEEFT can be deduced from the vanishing of the tree-level L-point correlator $\psi \psi \phi^{\mathrm{L}-3}$. It is easy to see that the vertex operators $V_{-1}$ and $V_{-1 / 2}$ corresponding to the bosonic and fermionic fields in a left chiral supermultiplet are related by a shift in their fermionic weights

$$
q_{s h}^{(1)}=q_{s h}^{(1 / 2)}+\left( \pm \frac{1}{2}, \pm \frac{1}{2},-\frac{1}{2},-\frac{1}{2},-\frac{1}{2}\right)
$$


The computation of the tree-level amplitude requires the emission vertices to cancel the background ghost-charge of two on the sphere. Thus it is necessary to shift the ghost picture of some of the vertex operators according to

$$
V_{0}=e^{\phi} T_{\mathrm{F}} V_{-1}
$$

where $T_{\mathrm{F}}$ is the worldsheet supersymmetry current $[38,39]$

$$
T_{\mathrm{F}}=\bar{\partial} X^{i} \bar{\psi}^{i}+\bar{\partial} \bar{X}^{i} \psi^{i},
$$

with $\psi^{j}=\exp \left\{\mathrm{i} q_{j} \cdot H\right\}$ and $q_{j}^{i}=\delta_{j}^{i}$. This picture-changing operation allows one to write bosonic vertices with zero ghost-charge, at the price of introducing the right-moving oscillators $\bar{\partial} X^{i}$ and $\bar{\partial} \bar{X}^{i}$ and additional H-momentum. The correlator can then be written as

$$
\mathcal{F}=\left\langle V_{-1 / 2}\left(z_{1}, \bar{z}_{1}\right) V_{-1 / 2}\left(z_{2}, \bar{z}_{2}\right) V_{-1}\left(z_{3}, \bar{z}_{3}\right) V_{0}\left(z_{4}, \bar{z}_{4}\right) \ldots V_{0}\left(z_{\mathrm{L}}, \bar{z}_{\mathrm{L}}\right)\right\rangle,
$$

where each $V_{\alpha}=V\left(z_{\alpha}, \bar{z}_{\alpha}\right)$ represents a certain physical state from the massless spectrum. It is possible to infer several selection rules from the explicit form of the correlator. ${ }^{2}$ In the following we will make use of the space group selection rule

$$
\mathbb{1} \subset \prod_{\alpha=1}^{\mathrm{L}}\left[g_{\alpha}\right]
$$

gauge invariance

$$
\sum_{\alpha=1}^{\mathrm{L}} p_{s h \alpha}^{I}=0
$$

and H-momentum conservation

$$
\sum_{\alpha=1}^{\mathrm{L}} q_{\text {sh } \alpha}^{(1) i}=-1-\mathcal{N}_{R}^{i}
$$

where $\mathcal{N}_{R}^{i}$ counts the number of holomorphic right-moving oscillators in the correlator. Using the above rules the correlator (3.4) can be rewritten in the form

$$
\mathcal{F}=\left\langle\prod_{\alpha=1}^{\mathrm{L}}\left(\prod_{i=1}^{3}\left(\partial X^{i}\right)^{\mathcal{N}_{L}^{i} \alpha}\left(\partial \bar{X}^{i}\right)^{\overline{\mathcal{N}}_{L}^{i} \alpha}\left(\bar{\partial} X^{i}\right)^{\mathcal{N}_{R}^{i}}\right) \sigma_{\alpha}\right\rangle .
$$

Let us now deduce the coupling selection rules arising from symmetries of the orbifold geometries. A classification of these symmetries was drawn in the appendix of [20]. In particular, we are interested in rotations of the torus lattice, which leave the fixed-point structure of the orbifold invariant. This subgroup of automorphisms was called $D$ in [20]. Denoting these automorphisms by $\varrho$, they satisfy

$$
\theta=\varrho \theta \varrho^{-1} \quad \text { and } \quad \varrho(g) \in[g] \forall g \in S,
$$

\footnotetext{
${ }^{2}$ For a review on these selection rules we refer to [40].
} 
and in general, take a block diagonal form, i.e.

$$
\varrho=\operatorname{diag}\left(e^{2 \pi \mathrm{i} \xi^{1}}, e^{2 \pi \mathrm{i} \xi^{2}}, e^{2 \pi \mathrm{i} \xi^{3}}\right) .
$$

By definition, given a $g \in S, \varrho(g)$ is conjugate to $g$, and hence there exists a space group element $h_{g}$ such that

$$
\varrho(g)=h_{g} g h_{g}^{-1} .
$$

Writing $g=\left(\theta^{k}, \lambda\right)$ and $\varrho(g)=\left(\theta^{k}, \varrho \lambda\right), h_{g}=\left(\theta^{l}, \mu\right)$ can be determined by finding a solution to the equation

$$
\mu=\left(1-\theta^{k}\right)^{-1}\left(\varrho-\theta^{l}\right) \lambda .
$$

In analogy to (2.10), the most general transformation behavior for the auxiliary twist fields under $\rho$ is given by

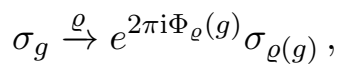

which, for the physical twist fields described in eq. (2.9), implies

$$
\sigma \stackrel{\varrho}{\rightarrow} \sum_{g^{\prime} \in[g]} e^{2 \pi \mathrm{i}\left[-\tilde{\gamma}\left(\varrho\left(g^{\prime}\right)\right)+\tilde{\gamma}\left(g^{\prime}\right)+\Phi_{\varrho}\left(g^{\prime}\right)\right]} e^{2 \pi \mathrm{i} \tilde{\gamma}\left(\varrho\left(g^{\prime}\right)\right)} \sigma_{\varrho\left(g^{\prime}\right)} .
$$

Since $\varrho$ preserves conjugacy classes, the vertex operators have to be invariant up to phases. This means that we have to require the structure of $\sigma$ to be preserved, which is guaranteed if the following condition is satisfied

$$
\tilde{\gamma}(g)-\tilde{\gamma}(\varrho(g))+\Phi_{\varrho}(g)=\tilde{\gamma}\left(h g h^{-1}\right)-\tilde{\gamma}\left(\varrho\left(h g h^{-1}\right)\right)+\Phi_{\varrho}\left(h g h^{-1}\right) \bmod 1 .
$$

Using $\varrho\left(h g h^{-1}\right)=\varrho(h) \varrho(g) \varrho(h)^{-1}$ and the definition (2.12) we find

$$
\Phi_{\varrho}\left(h g h^{-1}\right)=\Phi_{\varrho}(g)+\gamma_{h}-\gamma_{\varrho(h)} \bmod 1 .
$$

Note that this equation implies that once we know the phase $\Phi_{\varrho}(g)$, the phases for all other elements of the conjugacy class are automatically fixed. Note moreover that the phase, $\Phi_{\varrho}(g)$, acquired by the auxiliary twist field $\sigma_{g}$ must depend only on the space-group element $g$, whereas the gamma-phases associated with the physical twist field $\sigma$ depend, via (2.16), on the quantum numbers of the corresponding state. Therefore, if (3.16) is to be fulfilled for all physical states, the vacuum-phases and gamma-phases must independently fulfill

$$
\begin{aligned}
\Phi_{\varrho}\left(h g h^{-1}\right)-\Phi_{\varrho}(g) & =0 \bmod 1 \\
\gamma_{h}-\gamma_{\varrho(h)} & =0 \bmod 1
\end{aligned}
$$

for all space group elements $g, h$. Now, plugging (3.11) into (3.15) and using (3.17) we find

$$
\gamma_{h_{g}}=\gamma_{h_{g^{\prime}}} \bmod 1
$$

for all $g^{\prime} \in[g]$. This permits the transformation of the $\sigma$ twist to be recast to the desired form

$$
\sigma \stackrel{\varrho}{\rightarrow} e^{2 \pi \mathrm{i}\left[\gamma_{h_{g}}+\Phi_{\varrho}(g)\right]} \sigma
$$


Note that we have left the phases $\Phi_{\varrho}(g)$ undetermined. Finally, the transformation behavior of the correlator (3.8) under $\varrho$ can be concluded. It follows that it is only invariant in the case

$$
\sum_{i} \xi^{i}\left(\sum_{\alpha=1}^{\mathrm{L}}\left(\mathcal{N}_{L \alpha}^{i}-\overline{\mathcal{N}}_{L}^{i} \alpha+\mathcal{N}_{R \alpha}^{i}\right)\right)+\sum_{\alpha=1}^{\mathrm{L}}\left(\gamma_{h_{g_{\alpha}}}+\Phi_{\varrho}\left(g_{\alpha}\right)\right)=0 \bmod 1 .
$$

The phases $\Phi_{\varrho}$ can be removed from the previous equation, since the space group selection rule together with the OPEs for the twist fields imply ${ }^{3}$

$$
\sum_{\alpha=1}^{\mathrm{L}} \Phi_{\varrho}\left(g_{\alpha}\right)=0 \bmod 1
$$

The invariance condition for the correlator can now be written in terms of well known quantities when combined with H-momentum conservation (3.7) and reads

$$
\sum_{\alpha=1}^{\mathrm{L}}\left(\sum_{i=1}^{3} \xi^{i}\left[q_{s h \alpha}^{(1) i}-\mathcal{N}_{L \alpha}^{i}+\overline{\mathcal{N}}_{L \alpha}^{i}\right]-\gamma_{h_{g_{\alpha}}}\right)=-\sum_{i=1}^{3} \xi^{i} \bmod 1 .
$$

In the case $\sum_{i} \xi^{i} \neq 0 \bmod 1$, this condition looks precisely like the coupling selection rule originating from an $R$-symmetry. In this case, take $M$ to be the smallest integer such that

$$
R \equiv-M \sum_{i} \xi^{i}
$$

is an integer. Then eq. (3.22) takes the more familiar form

$$
\sum_{\alpha=1}^{\mathrm{L}} r_{\alpha}=R \bmod M, \quad \text { with } \quad r_{\alpha}=\sum_{i=1}^{3} M \xi^{i}\left[q_{s h \alpha}^{(1) i}-\mathcal{N}_{L \alpha}^{i}+\overline{\mathcal{N}}_{L \alpha}^{i}\right]-M \gamma_{h_{g_{\alpha}}}
$$

Thus, by imposing the symmetry of the orbifold generated by $\varrho \in D$ on the correlation function, we have derived a quantity that can be readily interpreted as a $\mathbb{Z}_{M}^{R}$ discrete symmetry of the low energy effective field theory in which $R$ denotes the charge of the superpotential and $r_{\alpha}$ are the charges of the fields. ${ }^{4}$

Surprisingly, the discrete symmetry defined in (3.24) does not coincide with the explicit $R$-symmetry result derived in [20], due to the sign in the last term, which gives the contribution of the gamma-phase to the $R$-charges. In the following section we discuss this discrepancy in terms of the anomalies for both results.

\section{Universal $R$-symmetry anomalies}

In this section we compute the anomalies for the $\mathbb{Z}_{M}^{R}$-symmetry derived in the previous section and compare them with the result for the $\mathbb{Z}_{M}^{R^{\prime}}$-symmetry derived in [20]. In heterotic

\footnotetext{
${ }^{3}$ Using the space group selection rule, the leading term in the OPE of all auxiliary twist fields involved in the coupling is proportional to the identity, which transforms trivially under $\varrho$.

${ }^{4}$ For a comprehensive summary of $R$-charge conventions we refer to [41].
} 
orbifold compactifications, there is only one axion available to cancel would-be anomalies via the Green-Schwarz mechanism, so that one typically expects anomalies to be universal. Exceptions to this are the anomalies of discrete target-space modular symmetries, which in many cases can be made universal only after including contributions from one-loop threshold corrections [42, 43].

For anomalies involving U(1) factors the universality holds up to Kač-Moody levels, so we focus only on gravitational and non-Abelian anomalies for which the levels are all equal to 1 . Under a $\mathbb{Z}_{M}^{R}$ transformation the path integral measure transforms as

$$
\begin{aligned}
\mathcal{D} \psi \mathcal{D} \bar{\psi} \rightarrow \mathcal{D} \psi \mathcal{D} \bar{\psi} \exp \left[-2 \pi \mathrm{i} \frac{1}{M}\left(\sum_{a} A_{G_{a}^{2}-\mathbb{Z}_{M}^{R}} \cdot \frac{1}{16 \pi^{2}} \int \operatorname{tr}\left\{\mathcal{F}_{a} \wedge \mathcal{F}_{a}\right\}\right.\right. & \\
& \left.\left.+A_{\text {grav. }^{2}-\mathbb{Z}_{M}^{R}} \cdot \frac{1}{284 \pi^{2}} \int \operatorname{tr}\{\mathcal{R} \wedge \mathcal{R}\}\right)\right],
\end{aligned}
$$

as can be seen from applying Fujikawa's method [44, 45], where the Pontryagin indices

$$
\frac{T\left(\mathbf{N}_{a}\right)}{16 \pi^{2}} \int \operatorname{tr}\left\{\mathcal{F}_{a} \wedge \mathcal{F}_{a}\right\} \quad \text { and } \quad \frac{1}{2} \frac{1}{284 \pi^{2}} \int \operatorname{tr}\{\mathcal{R} \wedge \mathcal{R}\}
$$

are integer valued $[46,47]$, and here $T\left(\mathbf{N}_{a}\right)$ denotes the Dynkin index of the fundamental representation. The corresponding anomaly coefficients are given by $[23-25,28,29]^{5}$

$$
\begin{aligned}
& A_{G_{a}^{2}-\mathbb{Z}_{M}^{R}}= C_{2}\left(G_{a}\right) \frac{R}{2}+\sum_{\alpha}\left(r_{\alpha}-\frac{R}{2}\right) T\left(\mathbf{R}_{a}^{\alpha}\right), \\
& A_{\text {grav. }{ }^{2}-\mathbb{Z}_{M}^{R}=}=\left(-21-1-N_{T}-N_{U}+\sum_{a} \operatorname{dim}\left\{\operatorname{adj}\left(G_{a}\right)\right\}\right) \frac{R}{2} \\
&+\sum_{\alpha}\left(r_{\alpha}-\frac{R}{2}\right) \cdot \operatorname{dim}\left\{\mathbf{R}^{\alpha}\right\},
\end{aligned}
$$

with $C_{2}\left(G_{a}\right)$ being the quadratic Casimir of $G_{a}, \alpha$ running over left chiral matter representations and $T\left(\mathbf{R}_{a}^{\alpha}\right)$ its corresponding Dynkin index. In eq. (4.4), the contributions of -21 and -1 correspond to the gravitino and dilatino respectively, $N_{T}$ and $N_{U}$ are the number of $T$ - and $U$-modulini and $a$ runs over all gauge factors (including $\mathrm{U}(1)$ 's). If anomalies are cancelled by the same axion shift, given two gauge factors $G_{a, b}$ the so-called universality conditions must hold

$$
\begin{aligned}
& A_{G_{a}^{2}-\mathbb{Z}_{M}^{R}} \bmod M T\left(\mathbf{N}_{a}\right)=A_{G_{b}^{2}-\mathbb{Z}_{M}^{R}} \bmod M T\left(\mathbf{N}_{b}\right), \\
& A_{G_{a}^{2}-\mathbb{Z}_{M}^{R}} \bmod M T\left(\mathbf{N}_{a}\right)=\frac{1}{24}\left(A_{\text {grav } .^{2}-\mathbb{Z}_{M}^{R}} \bmod \frac{M}{2}\right) .
\end{aligned}
$$

Let us focus on the orbifolds presented in table 1, with the isometries discussed in [20]. As examples, we give the space group elements $h_{g}$ needed to calculate the gamma-phases

\footnotetext{
${ }^{5}$ Recall that gauginos and matter fermions both contribute to the anomaly. The charge of the fermions can be inferred from the piece $\theta \psi \subset \Phi$ : if the charge of the multiplet $\Phi$ is denoted by $r$, then the charge of the fermion is $r-R / 2$. Analogously, the gauginos appear in the vector multiplet in the form $\overline{\theta \theta} \theta \lambda$, so that their charge is $R / 2$.
} 


\begin{tabular}{|c|c|c|c|c|c|c|c|}
\hline \multirow{2}{*}{ Orbifold } & \multirow{2}{*}{ Lattice } & \multirow{2}{*}{ Twist } & \multicolumn{3}{|c|}{$\varrho$} & \multirow{2}{*}{$R$} & \multirow{2}{*}{$M$} \\
\hline & & & $\xi^{1}$ & $\xi^{2}$ & $\xi^{3}$ & & \\
\hline \multirow{3}{*}{$\mathbb{Z}_{4}$} & \multirow{3}{*}{$\mathrm{SO}(4)^{2} \times \mathrm{SU}(2)^{2}$} & \multirow{3}{*}{$\left(\frac{1}{4}, \frac{1}{4},-\frac{2}{4}\right)$} & $1 / 4$ & $1 / 4$ & 0 & -1 & 2 \\
\hline & & & $1 / 2$ & 0 & 0 & -1 & 2 \\
\hline & & & 0 & 0 & $-1 / 2$ & +1 & 2 \\
\hline \multirow{2}{*}{$\mathbb{Z}_{4}$} & \multirow{2}{*}{$\mathrm{SU}(4)^{2}$} & \multirow{2}{*}{$\left(\frac{1}{4}, \frac{1}{4},-\frac{2}{4}\right)$} & $1 / 2$ & 0 & 0 & -1 & 2 \\
\hline & & & 0 & $1 / 2$ & 0 & -1 & 2 \\
\hline \multirow{2}{*}{$\mathbb{Z}_{6 I}$} & \multirow{2}{*}{$G_{2} \times G_{2} \times \mathrm{SU}(3)$} & \multirow{2}{*}{$\left(\frac{1}{6}, \frac{1}{6},-\frac{2}{6}\right)$} & $1 / 6$ & $1 / 6$ & 0 & -1 & 3 \\
\hline & & & 0 & 0 & $-1 / 3$ & +1 & 3 \\
\hline \multirow{3}{*}{$\mathbb{Z}_{6 I I}$} & \multirow{3}{*}{$G_{2} \times \mathrm{SU}(3) \times \mathrm{SU}(2)^{2}$} & \multirow{3}{*}{$\left(\frac{1}{6}, \frac{2}{6},-\frac{3}{6}\right)$} & $1 / 6$ & 0 & 0 & -1 & 6 \\
\hline & & & 0 & $1 / 3$ & 0 & -1 & 3 \\
\hline & & & 0 & 0 & $-1 / 2$ & +1 & 2 \\
\hline \multirow{2}{*}{$\mathbb{Z}_{8 I}$} & \multirow{2}{*}{$\mathrm{SO}(9) \times \mathrm{SO}(5)$} & \multirow{2}{*}{$\left(\frac{1}{8},-\frac{3}{8}, \frac{2}{8}\right)$} & $1 / 4$ & $-3 / 4$ & 0 & +1 & 2 \\
\hline & & & 0 & 0 & $1 / 2$ & -1 & 2 \\
\hline \multirow{2}{*}{$\mathbb{Z}_{8 I I}$} & \multirow{2}{*}{$\mathrm{SO}(8) \times \mathrm{SO}(4)$} & \multirow{2}{*}{$\left(\frac{1}{8}, \frac{3}{8},-\frac{4}{8}\right)$} & $1 / 8$ & $3 / 8$ & 0 & -1 & 2 \\
\hline & & & 0 & 0 & $-1 / 2$ & +1 & 2 \\
\hline \multirow{2}{*}{$\mathbb{Z}_{12 I}$} & \multirow{2}{*}{$\mathrm{SU}(3) \times F_{4}$} & \multirow{2}{*}{$\left(\frac{4}{12}, \frac{1}{12},-\frac{5}{12}\right)$} & $1 / 3$ & 0 & 0 & -1 & 3 \\
\hline & & & 0 & $1 / 12$ & $-5 / 12$ & +1 & 3 \\
\hline \multirow{2}{*}{$\mathbb{Z}_{12 I I}$} & \multirow{2}{*}{$F_{4} \times \mathrm{SO}(4)$} & \multirow{2}{*}{$\left(\frac{1}{12}, \frac{5}{12},-\frac{6}{12}\right)$} & $1 / 12$ & $5 / 12$ & 0 & -1 & 2 \\
\hline & & & 0 & 0 & $-1 / 2$ & +1 & 2 \\
\hline
\end{tabular}

Table 1. Summary of point groups studied with their corresponding lattices and corresponding orbifold isometries. The charge of the superpotential $R$ and the order of the symmetry $M$ are also given.

for the $R$-symmetries identified for the $\mathbb{Z}_{4}$ and $\mathbb{Z}_{6 I I}$ orbifold in the appendix. We used the $\mathrm{C}++$ orbifolder [48] to compute the spectrum and the corresponding anomalies for all of the embeddings classified in $[49,51]$ without Wilson lines, with the $R$-charge assignment given in eq. (3.24) for the factorizable $\mathbb{Z}_{4}, \mathbb{Z}_{6 I}$ and $\mathbb{Z}_{6 I I}$, as well as the non-factorizable $\mathbb{Z}_{8 I}$ orbifold. In all models the $R$-anomalies satisfy universality conditions. When taking the $R$-charges without the gamma contribution [6], universality is particular to very few models. The same is observed when using the opposite sign for the $\gamma$ phases, as derived in [20]. Furthermore we considered models with Wilson lines. For each of the allowed shift embeddings we randomly generated 10000 Wilson line configurations and found that in these cases the $R$ charges computed from eq. (3.24) show universality relations for all orbifolds studied. This is an overwhelming result and a strong hint that the $R$-charges derived here are correct. However, the reason for the opposite sign in front of the gamma-phase contribution derived in [20] remains to be understood. Here, we discuss a possible way out. We have assumed the auxiliary twist fields $\sigma_{g}$ to transform according to (2.10). Suppose instead that the 
auxiliary twist fields $\sigma_{g}$ have the (albeit counter-intuitive) transformation law

$$
\sigma_{g} \stackrel{h}{\rightarrow} e^{2 \pi \mathrm{i} \Phi(g, h)} \sigma_{h^{-1} g h},
$$

where the role of $h$ and $h^{-1}$ is interchanged compared to (2.10). The resulting $R$-charge assignment (3.22), (3.24) is independent of this change. Indeed, if one goes through the derivations in section 3 using this transformation behavior one arrives at

$$
\sigma \stackrel{\varrho}{\rightarrow} e^{2 \pi \mathrm{i}\left[\gamma_{h_{g}^{-1}}+\Phi_{\varrho}(g)\right]} \sigma
$$

instead of (3.19), while (2.16) gets modified to

$$
\gamma_{h}=p_{s h} \cdot V_{h}-v_{h}^{i}\left(q_{s h}^{(a) i}-\mathcal{N}_{L}^{i}+\overline{\mathcal{N}}_{L}^{i}\right)+\Phi(g, h),
$$

so that the resulting $R$-charge conservation law remains precisely (3.24). Meanwhile, the same $R$-charge conservation law (3.24) would be derived by the explicit computations in $[20]$.

\section{$5 \quad$ Further $R$-symmetry candidates}

Having observed universality for the $R$-symmetries derived above, let us now elaborate on an additional set of symmetries which was already introduced in [20]. There we observed that some lattice automorphisms exchange certain fixed points of the same twisted sector. We denoted the subgroup of lattice automorphisms satisfying this property by $F$. At first it seems that this kind of isometry has nothing to do with the string orbifold compactification. In some cases, however, one observes that the fixed points which get mapped to each other under a certain $\zeta \in F$ allocate identical matter representations, and hence it gives rise to a symmetry in the low energy effective field theory. ${ }^{6}$

Now we are concerned with the computation of the charges of the fields under this new type of symmetry. We will use the fact that, for all cases considered, the elements in $F$ can be written in a block diagonal form as

$$
\zeta=\operatorname{diag}\left(e^{2 \pi \mathrm{i} \eta^{1}}, e^{2 \pi \mathrm{i} \eta^{2}}, e^{2 \pi \mathrm{i} \eta^{3}}\right) .
$$

As expected, for those vertex operators which are eigenstates of $\zeta$, the charges are similar to $(3.24)$ :

$$
r_{\alpha}=\sum_{i=1}^{3} M \eta^{i}\left[q_{s h \alpha}^{(a) i}-\mathcal{N}_{L \alpha}^{i}+\overline{\mathcal{N}}_{L \alpha}^{i}\right]-M \gamma_{h_{g_{\alpha}}} .
$$

Let us therefore consider vertices of states located at non-invariant fixed points. For simplicity, let us assume that $\zeta$ only interchanges certain conjugacy classes, i.e.

$$
[g] \stackrel{\zeta}{\longleftrightarrow}\left[g^{\prime}\right], \quad g \nsim g^{\prime} .
$$

\footnotetext{
${ }^{6}$ Analogous symmetries were considered in [52], but as these were permutation symmetries rather than rotational symmetries, they did not correspond to $R$-symmetries.
} 
This implies that a vertex $V$ from $[g]$ gets mapped to its counterpart $V^{\prime}$, where $V$ and $V^{\prime}$ share the same quantum numbers. ${ }^{7}$ Writing

$$
\begin{aligned}
\sigma & \sim \sum_{g \in[g]} e^{2 \pi \mathrm{i} \tilde{\gamma}(g)} \sigma_{g}, \\
\sigma^{\prime} & \sim \sum_{g^{\prime} \in\left[g^{\prime}\right]} e^{2 \pi \mathrm{i} \tilde{\gamma}^{\prime}\left(g^{\prime}\right)} \sigma_{g^{\prime}},
\end{aligned}
$$

the twist fields involved in these vertices will then transform according to ${ }^{8}$

$$
\begin{gathered}
\sigma \stackrel{\zeta}{\rightarrow} \exp \left\{2 \pi \mathrm{i}\left[\tilde{\gamma}(g)-\tilde{\gamma}^{\prime}(\zeta(g))+\Phi_{\zeta}(g)\right]\right\} \sigma^{\prime}, \\
\sigma^{\prime} \stackrel{\zeta}{\rightarrow} \exp \left\{2 \pi \mathrm{i}\left[\tilde{\gamma}^{\prime}\left(g^{\prime}\right)-\tilde{\gamma}\left(\zeta\left(g^{\prime}\right)\right)+\Phi_{\zeta}\left(g^{\prime}\right)\right]\right\} \sigma,
\end{gathered}
$$

and therefore

$$
\begin{gathered}
V \stackrel{\zeta}{\rightarrow} \exp \left\{2 \pi \mathrm{i}\left[-\eta^{i}\left(q_{s h}^{(a) i}-\mathcal{N}_{L}^{i}+\overline{\mathcal{N}}_{L}^{i}\right)+\tilde{\gamma}(g)-\tilde{\gamma}^{\prime}(\zeta(g))+\Phi_{\zeta}(g)\right]\right\} V^{\prime}, \\
V^{\prime} \stackrel{\zeta}{\rightarrow} \exp \left\{2 \pi \mathrm{i}\left[-\eta^{i}\left(q_{s h}^{(a) i}-\mathcal{N}_{L}^{i}+\overline{\mathcal{N}}_{L}^{i}\right)+\tilde{\gamma}^{\prime}\left(g^{\prime}\right)-\tilde{\gamma}\left(\zeta\left(g^{\prime}\right)\right)+\Phi_{\zeta}\left(g^{\prime}\right)\right]\right\} V
\end{gathered}
$$

Recall that $q_{s h}^{(a) i}, \mathcal{N}_{L}^{i}$ and $\overline{\mathcal{N}}_{L}^{i}$ are the same for both $V$ and $V^{\prime}$. Note that, a priori, the transformation phases in (5.4), (5.5) cannot be related to physical gamma-phases since $g$ and $\zeta(g)$ belong to different conjugacy classes. Since $V$ and $V^{\prime}$ differ only in their conjugacy classes and carry identical quantum numbers, one expects couplings involving either $V$ and $V^{\prime}$ to differ only by constant phases. One can write the vertices $V$ and $V^{\prime}$ in a basis of eigenstates of $\zeta$

$$
V^{(s)}=V+e^{2 \pi \mathrm{i}(\delta+s)} V^{\prime}, \quad s=0, \frac{1}{2},
$$

in which $\delta$ is a phase fixed so that the operators $V^{(s)}$ transform indeed only up to a phase under $\zeta$. Using equations (5.5) and (5.6), we can fix $\delta$ and write the transformation behavior of $V^{(s)}$ under $\zeta$ as

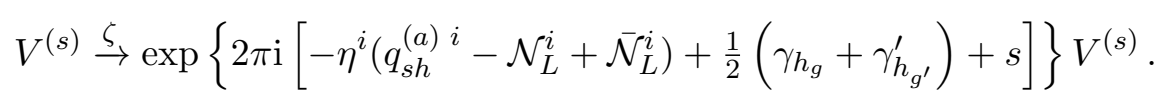

Here the space group elements $h_{g}$ and $h_{g^{\prime}}$ are defined such that

$$
\zeta\left(g^{\prime}\right)=h_{g} g h_{g}^{-1}, \quad \zeta(g)=h_{g^{\prime}} g^{\prime} h_{g^{\prime}}^{-1},
$$

for any combination of representatives $g$ and $g^{\prime}$ in the same way as described in section 3, and recall that $\gamma_{h_{g}}=\tilde{\gamma}(g)-\tilde{\gamma}\left(h_{g} g h_{g}^{-1}\right)$ and $\gamma_{h_{g^{\prime}}}^{\prime}=\tilde{\gamma}^{\prime}\left(g^{\prime}\right)-\tilde{\gamma}^{\prime}\left(h_{g^{\prime}} g^{\prime} h_{g^{\prime}}^{-1}\right)$. From the transformation property of the $V^{(s)}$ we can now read off their corresponding $R$-charges

$$
r^{(s)}=M \sum_{i=1}^{3} \eta^{i}\left(q_{s h}^{(a) i}-\mathcal{N}_{L}^{i}+\overline{\mathcal{N}}_{L}^{i}\right)-\frac{1}{2} M\left(\gamma_{h_{g}}+\gamma_{h_{g^{\prime}}}^{\prime}\right)-M s,
$$

\footnotetext{
${ }^{7}$ Note that although $V$ and $V^{\prime}$ are associated with different conjugacy classes, the conjugacy class is not a good quantum number to distinguish the states. Moreover, note that if the coupling $\left\langle V_{1} \ldots V_{\mathrm{L}-1} V\right\rangle$ is allowed by all selection rules, then so is $\left\langle V_{1} \ldots V_{\mathrm{L}-1} V^{\prime}\right\rangle$.

${ }^{8}$ In general one can allow for vacuum phases for the twist fields under this transformation. However they turn out to be irrelevant for our discussion in the same way as already observed in section 3.
} 
where $M$ is the smallest integer such that

$$
R \equiv-M \sum_{i=1}^{3} \eta^{i} \in \mathbb{Z} .
$$

When writing the low energy effective field theory in terms of the $\zeta$-eigenstates, the corresponding R-charge conservation law implies that any coupling must vanish unless

$$
\sum_{\alpha=1}^{\mathrm{L}} r_{\alpha}=R \bmod M .
$$

The result we just obtained for the elements in $F$, at least for the case of factorizable orbifolds, has remarkable implications. $R$-symmetries of the LEEFT are not only due to those remnants of the Lorentz group which leave the fixed points invariant. Even those automorphisms mapping different fixed point conjugacy classes to each other can source $R$ symmetries in the field theory. In contrast to those emerging from symmetries in $D$, these novel $R$-symmetries can be broken by Wilson line configurations that spoil the degeneracy of the states located at the non-invariant fixed points. Note that in our derivation we assumed that $\zeta$ at most interchanges pairs of conjugacy classes, but in principle more intricate transformation patterns can emerge, particularly in the case of non-factorizable orbifolds. We expect that in those cases, the charges can be computed in a similar fashion.

\section{An explicit example: $\mathbb{Z}_{4}$ on $\mathrm{SO}(4)^{2} \times \mathrm{SU}(2)^{2}$}

Here we illustrate our results by discussing in detail the $\mathbb{Z}_{4}$ orbifold on the lattice of $\mathrm{SO}(4)^{2} \times \mathrm{SU}(2)^{2}$ with the twist as given in table 1 . One easily sees that a basis of generators for the group $D$ is given by

$$
\begin{aligned}
& \varrho_{1}=\theta_{1} \theta_{2}=\left(e^{2 \pi \mathrm{i} \frac{1}{4}}, e^{2 \pi \mathrm{i} \frac{1}{4}}, 1\right), \quad \varrho_{2}=\left(\theta_{1}\right)^{2}=\left(e^{2 \pi \mathrm{i} \frac{1}{2}}, 1,1\right), \\
& \varrho_{3}=\theta_{3}=\left(1,1, e^{-2 \pi \mathrm{i} \frac{1}{4}}\right) .
\end{aligned}
$$

As stressed before, each of these symmetries leads to universal anomalies in all of the models studied. As an example let us discuss the following shift embedding and Wilson line configuration

$$
\begin{aligned}
V & =\left(-1,-\frac{3}{4}, 0,0,0,0,0, \frac{1}{4}, 0,0,0,0,0,0,0, \frac{1}{2}\right) \\
W_{1}=W_{2} & =\left(\frac{7}{4}, \frac{1}{4},-\frac{3}{4},-\frac{1}{4}, \frac{1}{4}, \frac{1}{4}, \frac{5}{4}, \frac{1}{4},-\frac{7}{4},-\frac{1}{4},-\frac{1}{4},-\frac{1}{4},-\frac{1}{4},-\frac{1}{4}, \frac{1}{4}, \frac{7}{4}\right), \\
W_{3}=W_{4} & =\left(-\frac{1}{2},-\frac{3}{2},-\frac{3}{2}, 1,-\frac{3}{2}, \frac{3}{2}, \frac{3}{2}, 1,-\frac{1}{4},-\frac{7}{4},-\frac{5}{4},-\frac{1}{4}, \frac{1}{4}, \frac{1}{4}, \frac{3}{4},-\frac{7}{4}\right), \\
W_{5} & =\left(0,-\frac{1}{2}, \frac{3}{2},-\frac{3}{2}, \frac{3}{2}, \frac{3}{2}, 1, \frac{3}{2}, 1,-2,0,1, \frac{1}{2}, 2,1,-\frac{3}{2}\right), \\
W_{6} & =0
\end{aligned}
$$



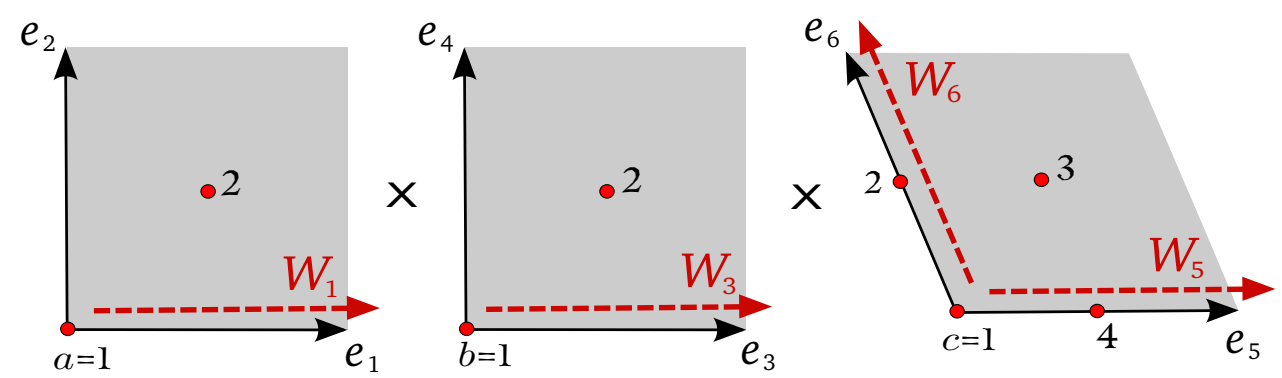

Figure 1. Wilson line configuration for the $\mathbb{Z}_{4}$ orbifold studied in the text.

Recall the identifications for the Wilson lines: $W_{1} \sim W_{2}, W_{3} \sim W_{4}$, see figure 1 where the Wilson line configuration for this model is shown.

This embedding leaves the following gauge symmetry unbroken

$$
\mathrm{SU}(4)_{1} \times \mathrm{SU}(2)_{1} \times \mathrm{SU}(2)_{2} \times \mathrm{SU}(4)_{2} \times \mathrm{SU}(2)_{3} \times \mathrm{U}(1)^{7} \subset E_{8} \times E_{8} .
$$

The anomaly coefficients obtained for this specific orbifold model are

$$
\begin{aligned}
& A_{\text {grav. }}{ }^{2} \varrho_{1}=-76, \quad A_{\text {grav. }}{ }^{2} \varrho_{2}=94, \quad A_{\text {grav. }^{2}{ }^{2} \varrho_{3}}=84, \\
& A_{\mathrm{SU}(4)_{1}^{2}-\varrho_{1}}=-3, \quad A_{\mathrm{SU}(4)_{1}^{2}-\varrho_{2}}=3, \quad A_{\mathrm{SU}(4)_{1}^{2}-\varrho_{3}}=-1, \\
& A_{\mathrm{SU}(2)_{1}^{2}-\varrho_{1}}=-5, \quad A_{\mathrm{SU}(2)_{1}^{2}-\varrho_{2}}=1, \quad A_{\mathrm{SU}(2)_{1}^{2}-\varrho_{3}}=5, \\
& A_{\mathrm{SU}(2) 2}-\varrho_{1}=-11, \quad A_{\mathrm{SU}(2){ }_{2}^{2}-\varrho_{2}}=6, \quad A_{\mathrm{SU}(2)_{2}^{2}-\varrho_{3}}=5, \\
& A_{\mathrm{SU}(4)_{2}^{2}-\varrho_{1}}=-3, \quad A_{\mathrm{SU}(4)_{2}^{2}-\varrho_{2}}=-1, \quad A_{\mathrm{SU}(4)_{2}^{2}-\varrho_{3}}=-1, \\
& \left.A_{\mathrm{SU}(2)}\right)_{3}-\varrho_{1}=-11, \quad A_{\mathrm{SU}(2)_{3}^{2}-\varrho_{2}}=3, \quad A_{\mathrm{SU}(2)_{3}^{2}-\varrho_{3}}=5 .
\end{aligned}
$$

One can straightforwardly check that all of these values satisfy the universality conditions (4.5) and (4.6).

This model also serves to discuss the effects of the new $R$-symmetries emerging from $F$. Note that

$$
\zeta=\theta_{1}=\left(e^{2 \pi \mathrm{i} \frac{1}{4}}, 1,1\right) \in F
$$

interchanges the fixed points

$$
z_{g}=\frac{e_{2}+e_{3}}{2} \stackrel{\zeta}{\longleftrightarrow} z_{g^{\prime}}=\frac{e_{2}+e_{4}}{2},
$$

of the second twisted sector $T_{2}$, which are generated by space group elements $g$ and $g^{\prime}$ from different conjugacy classes. This is illustrated in figure 2.

Note that as we have $W_{1}=W_{2}, W_{3}=W_{4}$, the transformation $\zeta$ respects the Wilson line structure. Hence the spectrum contains identical states $V$ and $V^{\prime}$ sitting at each of the relevant fixed points. As an example consider the states specified by the following quantum numbers

$$
\begin{aligned}
p_{s h} & =\left(-\frac{3}{4}, \frac{1}{4},-\frac{1}{4},-\frac{1}{4},-\frac{1}{4},-\frac{1}{4},-\frac{1}{4},-\frac{1}{4}, 0,0,-\frac{1}{2}, \frac{1}{2}, 0,0,0,0\right), \\
q_{s h} & =\left(0,-\frac{1}{2},-\frac{1}{2}, 0\right)
\end{aligned}
$$




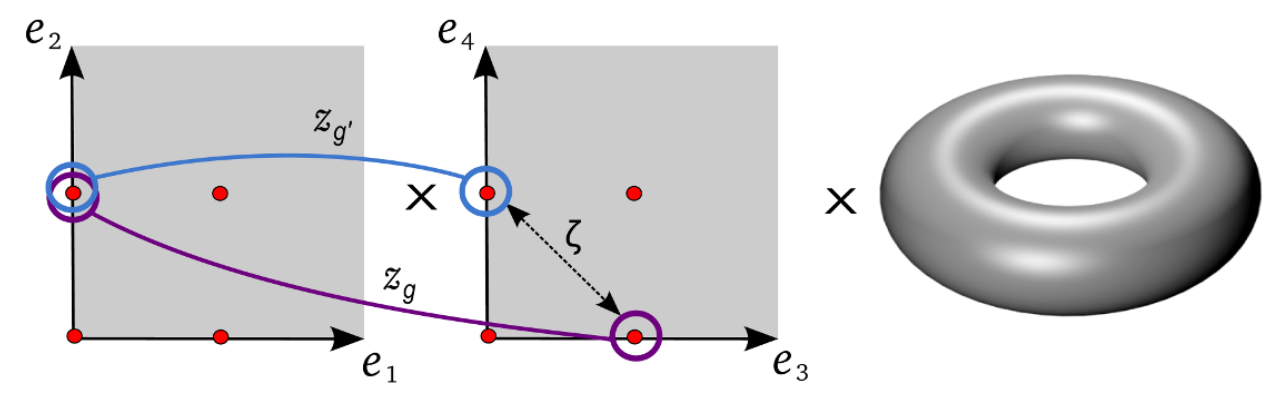

Figure 2. Representation of the $\theta_{1}$ action on the $T_{2}$ sector fixed points of the $\mathbb{Z}_{4}$ orbifold studied.

with no left-moving oscillators. The $p_{s h}$ presented is the highest weight of the representation $(\mathbf{1}, \mathbf{2}, \mathbf{2}, \mathbf{1}, \mathbf{2})$ with all $\mathrm{U}(1)$ charges equal to zero. Two identical copies of this state live at the fixed points under consideration. The elements $h_{g}$ and $h_{g^{\prime}}$ needed to compute the $R$-charges are given by

$$
h_{g}=\left(\theta, e_{3}\right) \quad \text { and } \quad h_{g^{\prime}}=(\theta, 0),
$$

and the corresponding gamma-phases are $\gamma(g)=\gamma\left(g^{\prime}\right)=3 / 4$. With this information we can compute the $R$-charges for the eigenstates of $\zeta$ to be

$$
r^{(s)}=-7 / 2-4 s .
$$

We also computed the anomaly coefficients for the $R$-symmetry $\zeta$, with a scan of over 100.000 randomly generated models. In all cases the anomalies turned out to be universal. Similar results are to be expected for all orbifolds for which the group $F$ is non-trivial. Note that, in our example, $\zeta^{2}=\varrho_{2}$ and one can show that the $R$-charges under $\varrho_{2}$ are twice those under $\zeta$ up to multiples of 2 . This implies that one can safely take $\zeta$ and $\varrho_{1}$ as a basis for all $R$-symmetries in the factorizable $\mathbb{Z}_{4}$ orbifold.

\section{Discussion}

In this work we have derived $R$-symmetries expected in the low energy effective field theory of heterotic orbifold compactifications, directly from the symmetries observed in the orbifold geometry. In particular, by imposing that the string correlation functions are invariant under such symmetries, we were able to infer the $R$-charges that are conserved in the low energy theory. This approach allowed us to be more general than the explicit computations of vanishing correlation functions pursued in [20]. For example, we were presently able to treat orbifold models with discrete Wilson lines. Moreover, we identified new $R$-symmetries, which arise from rotations which interchange inequivalent fixed points supporting the same physical states.

The conserved $R$-charges associated with rotations preserving the fixed point structure of the orbifold were derived in explicit models for all $\mathbb{Z}_{N}$ orbifold models, with and without discrete Wilson lines. The corresponding anomalies were then computed, and a scan of thousands of randomly generated models showed that the anomalies were universal. This is also the case for the $R$-symmetries conjectured for non-factorizable orbifolds, even though 
there are some non-trivial steps still missing for the full understanding of their CFT. Further, we identified an additional source for $R$-symmetries, namely those isometries under which certain fixed points (that support the same twisted matter) get exchanged. An example was given for a $\mathbb{Z}_{4}$ orbifold. It is remarkable that the corresponding $R$-symmetry anomalies were also found to satisfy universality relations.

The universality of the $R$-symmetry anomalies is certainly a beautiful and compelling result. On the other hand, the $R$-charges that were obtained from the explicit computation of vanishing string correlation functions [20], have the opposite sign in the gamma-phase contribution, and do not always lead to the universal anomalies. It remains an essential open question to understand the reason behind this mismatch, although we have pointed out a possible origin for the discrepancy. Moreover, it seems important to bear in mind the following observations. Anomaly universality does not necessarily guarantee that a symmetry is an exact symmetry. Examples in which anomalies are universal, but the symmetry is explicitly broken by non-perturbative effects, are some continuous targetspace modular symmetries [42, 53]. Meanwhile, anomalies which are non-universal might be partially cancelled by one-loop threshold effects, as sometimes observed for discrete target-space modular invariance [42, 43]. Finally, as discrete symmetries are by definition global there is no inconsistency if they happen to be anomalous. Simply, this would imply that they are not symmetries in the full quantum theory.

Despite the fact that the lattices studied here are the simplest possibilities, we expect similar results for the more general orbifold models discussed in [54-56]. It remains to be discussed how these redefined $R$-charges affect the phenomenology of MSSM like models found all over the orbifold landscape. In those models where the top Yukawa coupling is purely untwisted, one can guarantee its survival. However, in order to address issues such as Yukawa textures, decoupling of the exotics and proton decay it is necessary to look at explicit models. An important question concerns the effects of the new $R$-charge redefinitions in the particular context of $\mathbb{Z}_{2} \times \mathbb{Z}_{2}[17,18]$, especially in those models where the famous $\mathbb{Z}_{4}^{R}$ symmetry of $[57,58]$ could be realized. Another interesting issue has to do with the fact that the $R$-charges now receive contributions from the gauge part of the theory, so it is worth studying how the gauge bundle information enters the $R$-symmetries that one also expects to see in the orbifold phase of gauged linear sigma models, as well as in partial blow-ups.

\section{Acknowledgments}

We would like to thank M. Blaszczyk, S. Förste, P. Oehlmann and F. Rühle for useful discussions. N. G. C. B. is supported by "Proyecto Nacional de Ciencias Básicas Particulas y Campos" (CITMA, Cuba). T. K. is supported in part by the Grant-in-Aid for the Scientific Research No. 25400252 from the Ministry of Education, Culture, Sports, Science and Technology of Japan. S. L. P. is funded by Deutsche Forschungsgemeinschaft inside the "Graduiertenkolleg GRK 1463". The work of D. K. M. P. and M. S. was partially supported by the SFB-Tansregio TR33 "The Dark Universe" (Deutsche Forschungsgemeinschaft) and the European Union 7th network program "Unification in the LHC era" (PITN-GA-2009-237920). 


\section{A Space group elements $h_{g}$ for the $\mathbb{Z}_{4}$ and $Z_{6 I I}$ orbifolds}

In this appendix we present the values of $h_{g}$ for $\mathbb{Z}_{4}$ and $\mathbb{Z}_{6 I I}$ used in the main text, in tables 2 and 3 .

\begin{tabular}{|c|c|c|c|c|}
\hline$g$ & $h_{g}^{\theta_{1} \theta_{2}}$ & $h_{g}^{\theta_{3}}$ & $h_{g}^{\theta_{1}^{2}}$ & $h_{g}^{\theta_{1}}$ \\
\hline $\mathbb{1},(0,0,0,0,0,0)$ & $\mathbb{1},(0,0,0,0,0,0)$ & $\mathbb{1},(0,0,0,0,0,0)$ & $\mathbb{1},(0,0,0,0,0,0)$ & $\mathbb{1},(0,0,0,0,0,0)$ \\
\hline$\theta,(0,0,0,0,0,0)$ & $\mathbb{1},(0,0,0,0,0,0)$ & $\mathbb{1},(0,0,0,0,0,0)$ & $\mathbb{1},(0,0,0,0,0,0)$ & $\mathbb{1},(0,0,0,0,0,0)$ \\
\hline$\theta,(0,0,0,0,0,1)$ & $\mathbb{1},(0,0,0,0,0,0)$ & $\mathbb{1},(0,0,0,0,0,-1)$ & $\mathbb{1},(0,0,0,0,0,0)$ & $\mathbb{1},(0,0,0,0,0,0)$ \\
\hline$\theta,(0,0,1,0,0,0)$ & $\mathbb{1},(0,0,-1,0,0,0)$ & $\mathbb{1},(0,0,0,0,0,0)$ & $\mathbb{1},(0,0,0,0,0,0)$ & $\mathbb{1},(0,0,0,0,0,0)$ \\
\hline$\theta,(0,0,1,0,0,1)$ & $\mathbb{1},(0,0,-1,0,0,0)$ & $\mathbb{1},(0,0,0,0,0,-1)$ & $\mathbb{1},(0,0,0,0,0,0)$ & $\mathbb{1},(0,0,0,0,0,0)$ \\
\hline$\theta,(1,0,0,0,0,0)$ & $\mathbb{1},(-1,0,0,0,0,0)$ & $\mathbb{1},(0,0,0,0,0,0)$ & $\mathbb{1},(-1,-1,0,0,0,0)$ & $\mathbb{1},(-1,0,0,0,0,0)$ \\
\hline$\theta,(1,0,0,0,0,1)$ & $\mathbb{1},(-1,0,0,0,0,0)$ & $\mathbb{1},(0,0,0,0,0,-1)$ & $\mathbb{1},(-1,-1,0,0,0,0)$ & $\mathbb{1},(-1,0,0,0,0,0)$ \\
\hline$\theta,(1,0,1,0,0,0)$ & $\mathbb{1},(-1,0,-1,0,0,0)$ & $\mathbb{1},(0,0,0,0,0,0)$ & $\mathbb{1},(-1,-1,0,0,0,0)$ & $\mathbb{1},(-1,0,0,0,0,0)$ \\
\hline$\theta,(1,0,1,0,0,1)$ & $\mathbb{1},(-1,0,-1,0,0,0)$ & $\mathbb{1},(0,0,0,0,0,-1)$ & $\mathbb{1},(-1,-1,0,0,0,0)$ & $\mathbb{1},(-1,0,0,0,0,0)$ \\
\hline$\theta,(0,0,0,0,1,0)$ & $\mathbb{1},(0,0,0,0,0,0)$ & $\mathbb{1},(0,0,0,0,-1,0)$ & $\mathbb{1},(0,0,0,0,0,0)$ & $\mathbb{1},(0,0,0,0,0,0)$ \\
\hline$\theta,(0,0,0,0,1,1)$ & $\mathbb{1},(0,0,0,0,0,0)$ & $\mathbb{1},(0,0,0,0,-1,-1)$ & $\mathbb{1},(0,0,0,0,0,0)$ & $\mathbb{1},(0,0,0,0,0,0)$ \\
\hline$\theta,(0,0,1,0,1,0)$ & $\mathbb{1},(0,0,-1,0,0,0)$ &,$(0,0,0,0,-1,0)$ & $\mathbb{1},(0,0,0,0,0,0)$ & $\mathbb{1},(0,0,0,0,0,0)$ \\
\hline$\theta,(0,0,1,0,1,1)$ & $\mathbb{1},(0,0,-1,0,0,0)$ & $\mathbb{1},(0,0,0,0,-1,-1)$ & $\mathbb{1},(0,0,0,0,0,0)$ & $\mathbb{1},(0,0,0,0,0,0)$ \\
\hline$\theta,(1,0,0,0,1,0)$ & $\mathbb{1},(-1,0,0,0,0,0)$ & $\mathbb{1},(0,0,0,0,-1,0)$ & $\mathbb{1},(-1,-1,0,0,0,0)$ & $\mathbb{1},(-1,0,0,0,0,0)$ \\
\hline$\theta,(1,0,0,0,1,1)$ & $\mathbb{1},(-1,0,0,0,0,0)$ & $\mathbb{1},(0,0,0,0,-1,-1)$ & $\mathbb{1},(-1,-1,0,0,0,0)$ & $\mathbb{1},(-1,0,0,0,0,0)$ \\
\hline$\theta,(1,0,1,0,1,0)$ & $\mathbb{1},(-1,0,-1,0,0,0)$ & $\mathbb{1},(0,0,0,0,-1,0)$ & $\mathbb{1},(-1,-1,0,0,0,0)$ & $\mathbb{1},(-1,0,0,0,0,0)$ \\
\hline$\theta,(1,0,1,0,1,1)$ & $\mathbb{1},(-1,0,-1,0,0,0)$ & $\mathbb{1},(0,0,0,0,-1,-1)$ & $\mathbb{1},(-1,-1,0,0,0,0)$ & $\mathbb{1},(-1,0,0,0,0,0)$ \\
\hline$\theta^{2},(0,0,0,0,0,0)$ & $\mathbb{1},(0,0,0,0,0,0)$ & $\mathbb{1},(0,0,0,0,0,0)$ & $\mathbb{1},(0,0,0,0,0,0)$ & $\mathbb{1},(0,0,0,0,0,0)$ \\
\hline$\theta^{2},(0,0,0,1,0,0)$ & $\theta,(0,0,0,0,0,0)$ & $\mathbb{1},(0,0,0,0,0,0)$ & $\mathbb{1},(0,0,0,0,0,0)$ & $\mathbb{1},(0,0,0,0,0,0)$ \\
\hline$\theta^{2},(0,0,1,1,0,0)$ & $\mathbb{1},(0,0,-1,0,0,0)$ & $\mathbb{1},(0,0,0,0,0,0)$ & $\mathbb{1},(0,0,0,0,0,0)$ & $\mathbb{1},(0,0,0,0,0,0)$ \\
\hline$\theta^{2},(0,1,0,0,0,0)$ & $\theta,(0,0,0,0,0,0)$ & $\mathbb{1},(0,0,0,0,0,0)$ & $\mathbb{1},(0,-1,0,0,0,0)$ & $\theta,(0,0,0,0,0,0)$ \\
\hline$\theta^{2},(0,1,0,1,0,0)$ & $\theta,(0,0,0,0,0,0)$ & $\mathbb{1},(0,0,0,0,0,0)$ & $\mathbb{1},(0,-1,0,0,0,0)$ & $\theta,(0,0,0,0,0,0)^{\dagger}$ \\
\hline$\theta^{2},(0,1,1,0,0,0)$ & $\theta,(0,0,0,0,0,0)$ & $\mathbb{1},(0,0,0,0,0,0)$ & $\mathbb{1},(0,-1,0,0,0,0)$ & $\theta,(0,0,1,0,0,0)^{\dagger}$ \\
\hline$\theta^{2},(0,1,1,1,0,0)$ & $\theta,(0,0,0,0,0,0)$ & $\mathbb{1},(0,0,0,0,0,0)$ & $\mathbb{1},(0,-1,0,0,0,0)$ & $\theta,(0,0,1,0,0,0)$ \\
\hline$\theta^{2},(1,1,0,0,0,0)$ & $\mathbb{1},(-1,0,0,0,0,0)$ & $\mathbb{1},(0,0,0,0,0,0)$ & $\mathbb{1},(-1,-1,0,0,0,0)$ & $\mathbb{1},(-1,0,0,0,0,0)$ \\
\hline$\theta^{2},(1,1,0,1,0,0)$ & $\theta,(0,0,0,0,0,0)$ & $\mathbb{1},(0,0,0,0,0,0)$ & $\mathbb{1},(-1,-1,0,0,0,0)$ & $\mathbb{1},(-1,0,0,0,0,0)$ \\
\hline$\theta^{2},(1,1,1,1,0,0)$ & $\mathbb{1},(-1,0,-1,0,0,0)$ & $\mathbb{1},(0,0,0,0,0,0)$ & $\mathbb{1},(-1,-1,0,0,0,0)$ & $\mathbb{1},(-1,0,0,0,0,0)$ \\
\hline$\theta^{3},(0,0,0,0,0,0)$ & $\mathbb{1},(0,0,0,0,0,0)$ & $\mathbb{1},(0,0,0,0,0,0)$ & $\mathbb{1},(0,0,0,0,0,0)$ & $\mathbb{1},(0,0,0,0,0,0)$ \\
\hline$\theta^{3},(0,0,0,0,0,1)$ & $\mathbb{1},(0,0,0,0,0,0)$ & $\mathbb{1},(0,0,0,0,0,-1)$ & $\mathbb{1},(0,0,0,0,0,0)$ & $\mathbb{1},(0,0,0,0,0,0)$ \\
\hline$\theta^{3},(0,0,1,0,0,0)$ & $\mathbb{1},(0,0,0,1,0,0)$ & $\mathbb{1},(0,0,0,0,0,0)$ & $\mathbb{1},(0,0,0,0,0,0)$ & $\mathbb{1},(0,0,0,0,0,0)$ \\
\hline$\theta^{3},(0,0,1,0,0,1)$ & $\mathbb{1},(0,0,0,1,0,0)$ & $\mathbb{1},(0,0,0,0,0,-1)$ & $\mathbb{1},(0,0,0,0,0,0)$ & $\mathbb{1},(0,0,0,0,0,0)$ \\
\hline$\theta^{3},(1,0,0,0,0,0)$ & $\mathbb{1},(0,1,0,0,0,0)$ & $\mathbb{1},(0,0,0,0,0,0)$ & $\mathbb{1},(-1,1,0,0,0,0)$ & $\mathbb{1},(0,1,0,0,0,0)$ \\
\hline$\theta^{3},(1,0,0,0,0,1)$ & $\mathbb{1},(0,1,0,0,0,0)$ & $\mathbb{1},(0,0,0,0,0,-1)$ & $\mathbb{1},(-1,1,0,0,0,0)$ & $\mathbb{1},(0,1,0,0,0,0)$ \\
\hline$\theta^{3},(1,0,1,0,0,0)$ & $\mathbb{1},(0,1,0,1,0,0)$ & $\mathbb{1},(0,0,0,0,0,0)$ & $\mathbb{1},(-1,1,0,0,0,0)$ & $\mathbb{1},(0,1,0,0,0,0)$ \\
\hline$\theta^{3},(1,0,1,0,0,1)$ & $\mathbb{1},(0,1,0,1,0,0)$ & $\mathbb{1},(0,0,0,0,0,-1)$ & $\mathbb{1},(-1,1,0,0,0,0)$ & $\mathbb{1},(0,1,0,0,0,0)$ \\
\hline$\theta^{3},(0,0,0,0,1,0)$ & $\mathbb{1},(0,0,0,0,0,0)$ & $\mathbb{1},(0,0,0,0,-1,0)$ & $\mathbb{1},(0,0,0,0,0,0)$ & $\mathbb{1},(0,0,0,0,0,0)$ \\
\hline$\theta^{3},(0,0,0,0,1,1)$ & $\mathbb{1},(0,0,0,0,0,0)$ & $\mathbb{1},(0,0,0,0,-1,-1)$ & $\mathbb{1},(0,0,0,0,0,0)$ & $\mathbb{1},(0,0,0,0,0,0)$ \\
\hline$\theta^{3},(0,0,1,0,1,0)$ & $\mathbb{1},(0,0,0,1,0,0)$ & $\mathbb{1},(0,0,0,0,-1,0)$ & $\mathbb{1},(0,0,0,0,0,0)$ & $\mathbb{1},(0,0,0,0,0,0)$ \\
\hline$\theta^{3},(0,0,1,0,1,1)$ & $\mathbb{1},(0,0,0,1,0,0)$ & $\mathbb{1},(0,0,0,0,-1,-1)$ & $\mathbb{1},(0,0,0,0,0,0)$ & $\mathbb{1},(0,0,0,0,0,0)$ \\
\hline$\theta^{3},(1,0,0,0,1,0)$ & $\mathbb{1},(0,1,0,0,0,0)$ & $\mathbb{1},(0,0,0,0,-1,0)$ & $\mathbb{1},(-1,1,0,0,0,0)$ & $\mathbb{1},(0,1,0,0,0,0)$ \\
\hline$\theta^{3},(1,0,0,0,1,1)$ & $\mathbb{1},(0,1,0,0,0,0)$ & $\mathbb{1},(0,0,0,0,-1,-1)$ & $\mathbb{1},(-1,1,0,0,0,0)$ & $\mathbb{1},(0,1,0,0,0,0)$ \\
\hline$\theta^{3},(1,0,1,0,1,0)$ & $\mathbb{1},(0,1,0,1,0,0)$ & $\mathbb{1},(0,0,0,0,-1,0)$ & $\mathbb{1},(-1,1,0,0,0,0)$ & $\mathbb{1},(0,1,0,0,0,0)$ \\
\hline$\theta^{3},(1,0,1,0,1,1)$ & $\mathbb{1},(0,1,0,1,0,0)$ & $\mathbb{1},(0,0,0,0,-1,-1)$ & $\mathbb{1},(-1,1,0,0,0,0)$ & $\mathbb{1},(0,1,0,0,0,0)$ \\
\hline
\end{tabular}

Table 2. Values for $h_{g}$ 's for $\mathbb{Z}_{4}$. The elements marked with ${ }^{\dagger}$ correspond to the $h_{g}$ and $h_{g^{\prime}}$ from eq. (6.8). 


\begin{tabular}{|c|c|c|c|}
\hline$g$ & $h_{g}^{\theta_{1}}$ & $h_{g}^{\theta_{2}}$ & $h_{g}^{\theta_{3}}$ \\
\hline $\mathbb{1},(0,0,0,0,0,0)$ & $\mathbb{1},(0,0,0,0,0,0)$ & $\mathbb{1},(0,0,0,0,0,0)$ & $\mathbb{1},(0,0,0,0,0,0)$ \\
\hline$\theta,(0,0,1,1,1,1)$ & $\mathbb{1},(0,0,0,0,0,0)$ & $\mathbb{1},(0,0,-1,-1,0,0)$ & $\mathbb{1},(0,0,0,0,-1,-1)$ \\
\hline$\theta,(0,0,1,1,1,0)$ & $\mathbb{1},(0,0,0,0,0,0)$ & $\mathbb{1},(0,0,-1,-1,0,0)$ & $\mathbb{1},(0,0,0,0,-1,0)$ \\
\hline$\theta,(0,0,1,1,0,1)$ & $\mathbb{1},(0,0,0,0,0,0)$ & $\mathbb{1},(0,0,-1,-1,0,0)$ & $\mathbb{1},(0,0,0,0,0,-1)$ \\
\hline$\theta,(0,0,1,1,0,0)$ & $\mathbb{1},(0,0,0,0,0,0)$ & $\mathbb{1},(0,0,-1,-1,0,0)$ & $\mathbb{1},(0,0,0,0,0,0)$ \\
\hline$\theta,(0,0,0,0,1,1)$ & $\mathbb{1},(0,0,0,0,0,0)$ & $\mathbb{1},(0,0,0,0,0,0)$ & $\mathbb{1},(0,0,0,0,-1,-1)$ \\
\hline$\theta,(0,0,0,0,1,0)$ & $\mathbb{1},(0,0,0,0,0,0)$ & $\mathbb{1},(0,0,0,0,0,0)$ & $\mathbb{1},(0,0,0,0,-1,0)$ \\
\hline$\theta,(0,0,0,0,0,1)$ & $\mathbb{1},(0,0,0,0,0,0)$ & $\mathbb{1},(0,0,0,0,0,0)$ & $\mathbb{1},(0,0,0,0,0,-1)$ \\
\hline$\theta,(0,0,0,0,0,0)$ & $\mathbb{1},(0,0,0,0,0,0)$ & $\mathbb{1},(0,0,0,0,0,0)$ & $\mathbb{1},(0,0,0,0,0,0)$ \\
\hline$\theta,(0,0,1,0,1,1)$ & $\mathbb{1},(0,0,0,0,0,0)$ & $\mathbb{1},(0,0,-1,0,0,0)$ & $\mathbb{1},(0,0,0,0,-1,-1)$ \\
\hline$\theta,(0,0,1,0,1,0)$ & $\mathbb{1},(0,0,0,0,0,0)$ & $\mathbb{1},(0,0,-1,0,0,0)$ & $\mathbb{1},(0,0,0,0,-1,0)$ \\
\hline$\theta,(0,0,1,0,0,1)$ & $\mathbb{1},(0,0,0,0,0,0)$ & $\mathbb{1},(0,0,-1,0,0,0)$ & $\mathbb{1},(0,0,0,0,0,-1)$ \\
\hline$\theta,(0,0,1,0,0,0)$ & $\mathbb{1},(0,0,0,0,0,0)$ & $\mathbb{1},(0,0,-1,0,0,0)$ & $\mathbb{1},(0,0,0,0,0,0)$ \\
\hline$\theta^{2},(-1,1,0,2,0,0)$ & $\theta,(0,0,2,2,0,0)$ & $\mathbb{1},(0,0,-2,-2,0,0)$ & $\mathbb{1},(0,0,0,0,0,0)$ \\
\hline$\theta^{2},(-1,1,0,0,0,0)$ & $\theta,(0,0,0,0,0,0)$ & $\mathbb{1},(0,0,0,0,0,0)$ & $\mathbb{1},(0,0,0,0,0,0)$ \\
\hline$\theta^{2},(-1,1,0,1,0,0)$ & $\theta,(0,0,1,1,0,0)$ & $\mathbb{1},(0,0,-1,-1,0,0)$ & $\mathbb{1},(0,0,0,0,0,0)$ \\
\hline$\theta^{2},(0,0,0,2,0,0)$ & $\mathbb{1},(0,0,0,0,0,0)$ & $\mathbb{1},(0,0,-2,-2,0,0)$ & $\mathbb{1},(0,0,0,0,0,0)$ \\
\hline$\theta^{2},(0,0,0,0,0,0)$ & $\mathbb{1},(0,0,0,0,0,0)$ & $\mathbb{1},(0,0,0,0,0,0)$ & $\mathbb{1},(0,0,0,0,0,0)$ \\
\hline$\theta^{2},(0,0,0,1,0,0)$ & $\mathbb{1},(0,0,0,0,0,0)$ & $\mathbb{1},(0,0,-1,-1,0,0)$ & $\mathbb{1},(0,0,0,0,0,0)$ \\
\hline$\theta^{3},(1,0,0,0,1,1)$ & $\theta,(0,0,0,0,1,1)$ & $\mathbb{1},(0,0,0,0,0,0)$ & $\mathbb{1},(0,0,0,0,-1,-1)$ \\
\hline$\theta^{3},(1,0,0,0,1,0)$ & $\theta,(0,0,0,0,1,0)$ & $\mathbb{1},(0,0,0,0,0,0)$ & $\mathbb{1},(0,0,0,0,-1,0)$ \\
\hline$\theta^{3},(1,0,0,0,0,1)$ & $\theta,(0,0,0,0,0,1)$ & $\mathbb{1},(0,0,0,0,0,0)$ & $\mathbb{1},(0,0,0,0,0,-1)$ \\
\hline$\theta^{3},(1,0,0,0,0,0)$ & $\theta,(0,0,0,0,0,0)$ & $\mathbb{1},(0,0,0,0,0,0)$ & $\mathbb{1},(0,0,0,0,0,0)$ \\
\hline$\theta^{3},(0,0,0,0,1,1)$ & $\mathbb{1},(0,0,0,0,0,0)$ & $\mathbb{1},(0,0,0,0,0,0)$ & $\mathbb{1},(0,0,0,0,-1,-1)$ \\
\hline$\theta^{3},(0,0,0,0,1,0)$ & $\mathbb{1},(0,0,0,0,0,0)$ & $\mathbb{1},(0,0,0,0,0,0)$ & $\mathbb{1},(0,0,0,0,-1,0)$ \\
\hline$\theta^{3},(0,0,0,0,0,1)$ & $\mathbb{1},(0,0,0,0,0,0)$ & $\mathbb{1},(0,0,0,0,0,0)$ & $\mathbb{1},(0,0,0,0,0,-1)$ \\
\hline$\theta^{3},(0,0,0,0,0,0)$ & $\mathbb{1},(0,0,0,0,0,0)$ & $\mathbb{1},(0,0,0,0,0,0)$ & $\mathbb{1},(0,0,0,0,0,0)$ \\
\hline$\theta^{4},(-1,1,1,1,0,0)$ & $\theta,(0,0,1,1,0,0)$ & $\mathbb{1},(0,0,-1,-1,0,0)$ & $\mathbb{1},(0,0,0,0,0,0)$ \\
\hline$\theta^{4},(-1,1,0,0,0,0)$ & $\theta,(0,0,0,0,0,0)$ & $\mathbb{1},(0,0,0,0,0,0)$ & $\mathbb{1},(0,0,0,0,0,0)$ \\
\hline$\theta^{4},(-1,1,1,0,0,0)$ & $\theta,(0,0,1,0,0,0)$ & $\mathbb{1},(0,0,-1,0,0,0)$ & $\mathbb{1},(0,0,0,0,0,0)$ \\
\hline$\theta^{4},(0,0,1,1,0,0)$ & $\mathbb{1},(0,0,0,0,0,0)$ & $\mathbb{1},(0,0,-1,-1,0,0)$ & $\mathbb{1},(0,0,0,0,0,0)$ \\
\hline$\theta^{4},(0,0,0,0,0,0)$ & $\mathbb{1},(0,0,0,0,0,0)$ & $\mathbb{1},(0,0,0,0,0,0)$ & $\mathbb{1},(0,0,0,0,0,0)$ \\
\hline$\theta^{4},(0,0,1,0,0,0)$ & $\mathbb{1},(0,0,0,0,0,0)$ & $\mathbb{1},(0,0,-1,0,0,0)$ & $\mathbb{1},(0,0,0,0,0,0)$ \\
\hline$\theta^{5},(0,0,0,2,1,1)$ & $\mathbb{1},(0,0,0,0,0,0)$ & $\mathbb{1},(0,0,-2,-2,0,0)$ & $\mathbb{1},(0,0,0,0,-1,-1)$ \\
\hline$\theta^{5},(0,0,0,2,1,0)$ & $\mathbb{1},(0,0,0,0,0,0)$ & $\mathbb{1},(0,0,-2,-2,0,0)$ & $\mathbb{1},(0,0,0,0,-1,0)$ \\
\hline$\theta^{5},(0,0,0,2,0,1)$ & $\mathbb{1},(0,0,0,0,0,0)$ & $\mathbb{1},(0,0,-2,-2,0,0)$ & $\mathbb{1},(0,0,0,0,0,-1)$ \\
\hline$\theta^{5},(0,0,0,2,0,0)$ & $\mathbb{1},(0,0,0,0,0,0)$ & $\mathbb{1},(0,0,-2,-2,0,0)$ & $\mathbb{1},(0,0,0,0,0,0)$ \\
\hline$\theta^{5},(0,0,0,0,1,1)$ & $\mathbb{1},(0,0,0,0,0,0)$ & $\mathbb{1},(0,0,0,0,0,0)$ & $\mathbb{1},(0,0,0,0,-1,-1)$ \\
\hline$\theta^{5},(0,0,0,0,1,0)$ & $\mathbb{1},(0,0,0,0,0,0)$ & $\mathbb{1},(0,0,0,0,0,0)$ & $\mathbb{1},(0,0,0,0,-1,0)$ \\
\hline$\theta^{5},(0,0,0,0,0,1)$ & $\mathbb{1},(0,0,0,0,0,0)$ & $\mathbb{1},(0,0,0,0,0,0)$ & $\mathbb{1},(0,0,0,0,0,-1)$ \\
\hline$\theta^{5},(0,0,0,0,0,0)$ & $\mathbb{1},(0,0,0,0,0,0)$ & $\mathbb{1},(0,0,0,0,0,0)$ & $\mathbb{1},(0,0,0,0,0,0)$ \\
\hline$\theta^{5},(0,0,0,1,1,1)$ & $\mathbb{1},(0,0,0,0,0,0)$ & $\mathbb{1},(0,0,-1,-1,0,0)$ & $\mathbb{1},(0,0,0,0,-1,-1)$ \\
\hline$\theta^{5},(0,0,0,1,1,0)$ & $\mathbb{1},(0,0,0,0,0,0)$ & $\mathbb{1},(0,0,-1,-1,0,0)$ & $\mathbb{1},(0,0,0,0,-1,0)$ \\
\hline$\theta^{5},(0,0,0,1,0,1)$ & $\mathbb{1},(0,0,0,0,0,0)$ & $\mathbb{1},(0,0,-1,-1,0,0)$ & $\mathbb{1},(0,0,0,0,0,-1)$ \\
\hline$\theta^{5},(0,0,0,1,0,0)$ & $\mathbb{1},(0,0,0,0,0,0)$ & $\mathbb{1},(0,0,-1,-1,0,0)$ & $\mathbb{1},(0,0,0,0,0,0)$ \\
\hline
\end{tabular}

Table 3. Values for $h_{g}$ 's for $\mathbb{Z}_{6-I I}$. 
Open Access. This article is distributed under the terms of the Creative Commons Attribution License (CC-BY 4.0), which permits any use, distribution and reproduction in any medium, provided the original author(s) and source are credited.

\section{References}

[1] T. Banks, Effective lagrangian description of discrete gauge symmetries, Nucl. Phys. B 323 (1989) 90 [INSPIRE].

[2] J. Preskill and L.M. Krauss, Local discrete symmetry and quantum mechanical hair, Nucl. Phys. B 341 (1990) 50 [INSPIRE].

[3] M.G. Alford, S.R. Coleman and J. March-Russell, Disentangling non-abelian discrete quantum hair, Nucl. Phys. B 351 (1991) 735 [inSPIRE].

[4] L.J. Dixon, J.A. Harvey, C. Vafa and E. Witten, Strings on orbifolds, Nucl. Phys. B 261 (1985) 678 [INSPIRE].

[5] L.J. Dixon, J.A. Harvey, C. Vafa and E. Witten, Strings on orbifolds. 2, Nucl. Phys. B 274 (1986) 285 [INSPIRE].

[6] T. Kobayashi, S. Raby and R.-J. Zhang, Searching for realistic 4d string models with a Pati-Salam symmetry: orbifold grand unified theories from heterotic string compactification on a Z(6) orbifold, Nucl. Phys. B 704 (2005) 3 [hep-ph/0409098] [INSPIRE].

[7] T. Kobayashi, S. Raby and R.-J. Zhang, Constructing $5 D$ orbifold grand unified theories from heterotic strings, Phys. Lett. B 593 (2004) 262 [hep-ph/0403065] [INSPIRE].

[8] W. Buchmüller, K. Hamaguchi, O. Lebedev and M. Ratz, Supersymmetric standard model from the heterotic string, Phys. Rev. Lett. 96 (2006) 121602 [hep-ph/0511035] [INSPIRE].

[9] W. Buchmüller, K. Hamaguchi, O. Lebedev and M. Ratz, Supersymmetric standard model from the heterotic string (II), Nucl. Phys. B 785 (2007) 149 [hep-th/0606187] [INSPIRE].

[10] O. Lebedev et al., A mini-landscape of exact MSSM spectra in heterotic orbifolds, Phys. Lett. B 645 (2007) 88 [hep-th/0611095] [INSPIRE].

[11] O. Lebedev, H.P. Nilles, S. Ramos-Sanchez, M. Ratz and P.K. Vaudrevange, Heterotic mini-landscape. (II). Completing the search for MSSM vacua in a Z(6) orbifold, Phys. Lett. B 668 (2008) 331 [arXiv:0807.4384] [INSPIRE].

[12] S. Groot Nibbelink and O. Loukas, MSSM-like models on Z(8) toroidal orbifolds, JHEP 12 (2013) 044 [arXiv: 1308.5145] [INSPIRE].

[13] H. Kawabe, T. Kobayashi and N. Ohtsubo, Study of minimal string unification in Z(8) orbifold models, Phys. Lett. B 322 (1994) 331 [hep-th/9309069] [INSPIRE].

[14] J.E. Kim and B. Kyae, Flipped $\mathrm{SU}(5)$ from Z $(12-I)$ orbifold with Wilson line, Nucl. Phys. B 770 (2007) 47 [hep-th/0608086] [INSPIRE].

[15] J.E. Kim, J.-H. Kim and B. Kyae, Superstring standard model from Z(12 - I) orbifold compactification with and without exotics and effective R-parity, JHEP 06 (2007) 034 [hep-ph/0702278] [INSPIRE].

[16] J.E. Kim, Abelian discrete symmetries $\mathbb{Z}_{N}$ and $\mathbb{Z}_{n R}$ from string orbifolds, Phys. Lett. B 726 (2013) 450 [arXiv:1308.0344] [INSPIRE]. 
[17] M. Blaszczyk et al., $A Z_{2} \times Z_{2}$ standard model, Phys. Lett. B 683 (2010) 340 [arXiv:0911.4905] [INSPIRE].

[18] S. Förste, T. Kobayashi, H. Ohki and K.-j. Takahashi, Non-factorisable $Z_{2} \times Z_{2}$ heterotic orbifold models and Yukawa couplings, JHEP 03 (2007) 011 [hep-th/0612044] [INSPIRE].

[19] A. Font, L.E. Ibáñez, H.P. Nilles and F. Quevedo, On the concept of naturalness in string theories, Phys. Lett. B 213 (1988) 274 [INSPIRE].

[20] N.G. Cabo Bizet et al., $R$-charge conservation and more in factorizable and non-factorizable orbifolds, JHEP 05 (2013) 076 [arXiv:1301.2322] [INSPIRE].

[21] S. Hamidi and C. Vafa, Interactions on orbifolds, Nucl. Phys. B 279 (1987) 465 [INSPIRE].

[22] L.J. Dixon, D. Friedan, E.J. Martinec and S.H. Shenker, The conformal field theory of orbifolds, Nucl. Phys. B 282 (1987) 13 [INSPIRE].

[23] L.E. Ibáñez, More about discrete gauge anomalies, Nucl. Phys. B 398 (1993) 301 [hep-ph/9210211] [INSPIRE].

[24] L.E. Ibáñez and G.G. Ross, Discrete gauge symmetry anomalies, Phys. Lett. B 260 (1991) 291 [INSPIRE].

[25] T. Banks and M. Dine, Note on discrete gauge anomalies, Phys. Rev. D 45 (1992) 1424 [hep-th/9109045] [INSPIRE].

[26] M.B. Green and J.H. Schwarz, Anomaly cancellation in supersymmetric D $=10$ gauge theory and superstring theory, Phys. Lett. B 149 (1984) 117 [INSPIRE].

[27] T. Kobayashi and H. Nakano, 'Anomalous' U(1) symmetry in orbifold string models, Nucl. Phys. B 496 (1997) 103 [hep-th/9612066] [INSPIRE].

[28] T. Araki, K.-S. Choi, T. Kobayashi, J. Kubo and H. Ohki, Discrete R-symmetry anomalies in heterotic orbifold models, Phys. Rev. D 76 (2007) 066006 [arXiv:0705.3075] [InSPIRE].

[29] T. Araki et al., (Non-)Abelian discrete anomalies, Nucl. Phys. B 805 (2008) 124 [arXiv: 0805.0207] [INSPIRE].

[30] H.P. Nilles, S. Ramos-Sanchez, M. Ratz and P.K. Vaudrevange, A note on discrete $R$ symmetries in $\mathbb{Z}_{6}$-II orbifolds with Wilson lines, Phys. Lett. B 726 (2013) 876 [arXiv:1308.3435] [INSPIRE].

[31] L.E. Ibáñez, H.P. Nilles and F. Quevedo, Orbifolds and Wilson lines, Phys. Lett. B 187 (1987) 25 [INSPIRE].

[32] T. Kobayashi and N. Ohtsubo, Allowed Yukawa couplings of $Z_{N} \times Z_{M}$ orbifold models, Phys. Lett. B 262 (1991) 425 [INSPIRE].

[33] T. Kobayashi and N. Ohtsubo, Geometrical aspects of $Z_{N}$ orbifold phenomenology, Int. J. Mod. Phys. A 9 (1994) 87 [inSPIRE].

[34] F. Ploger, S. Ramos-Sanchez, M. Ratz and P.K. Vaudrevange, Mirage torsion, JHEP 04 (2007) 063 [hep-th/0702176] [INSPIRE].

[35] P. Goddard and D.I. Olive, Kac-Moody and Virasoro algebras in relation to quantum physics, Int. J. Mod. Phys. A 1 (1986) 303 [InSPIRE].

[36] T. Kobayashi and N. Ohtsubo, Yukawa coupling condition of $Z_{N}$ orbifold models, Phys. Lett. B 245 (1990) 441 [INSPIRE]. 
[37] J. Casas, F. Gómez and C. Muñoz, Complete structure of Z(n) Yukawa couplings, Int. J. Mod. Phys. A 8 (1993) 455 [hep-th/9110060] [INSPIRE].

[38] D. Friedan, E.J. Martinec and S.H. Shenker, Conformal invariance, supersymmetry and string theory, Nucl. Phys. B 271 (1986) 93 [INSPIRE].

[39] K.S. Choi and J.E. Kim, Quarks and leptons from orbifolded superstring, Lect. Notes Phys. volume 696, Springer, Berlin Germany (2006).

[40] T. Kobayashi, S.L. Parameswaran, S. Ramos-Sanchez and I. Zavala, Revisiting coupling selection rules in heterotic orbifold models, JHEP 05 (2012) 008 [Erratum ibid. 1212 (2012) 049] [arXiv: 1107.2137] [INSPIRE].

[41] C. Lüdeling, F. Ruehle and C. Wieck, Non-universal anomalies in heterotic string constructions, Phys. Rev. D 85 (2012) 106010 [arXiv:1203.5789] [INSPIRE].

[42] L.E. Ibáñez and D. Lüst, Duality anomaly cancellation, minimal string unification and the effective low-energy Lagrangian of $4 D$ strings, Nucl. Phys. B 382 (1992) 305 [hep-th/9202046] [INSPIRE].

[43] J. Derendinger, S. Ferrara, C. Kounnas and F. Zwirner, On loop corrections to string effective field theories: Field dependent gauge couplings and $\sigma$-model anomalies, Nucl. Phys. B 372 (1992) 145 [INSPIRE].

[44] K. Fujikawa, Path integral measure for gauge invariant fermion theories, Phys. Rev. Lett. 42 (1979) 1195 [inSPIRE].

[45] K. Fujikawa, Path integral for gauge theories with fermions, Phys. Rev. D 21 (1980) 2848 [Erratum ibid. D 22 (1980) 1499] [INSPIRE].

[46] L. Álvarez-Gaumé and E. Witten, Gravitational anomalies, Nucl. Phys. B 234 (1984) 269 [INSPIRE].

[47] L. Álvarez-Gaumé and P.H. Ginsparg, The structure of gauge and gravitational anomalies, Annals Phys. 161 (1985) 423 [Erratum ibid. 171 (1986) 233] [INSPIRE].

[48] H.P. Nilles, S. Ramos-Sanchez, P.K. Vaudrevange and A. Wingerter, The orbifolder: a tool to study the low energy effective theory of heterotic orbifolds, Comput. Phys. Commun. 183 (2012) 1363 [arXiv:1110.5229] [inSPIRE].

[49] Y. Katsuki, Y. Kawamura, T. Kobayashi, N. Ohtsubo and K. Tanioka, Gauge groups of $Z(n)$ orbifold models, Prog. Theor. Phys. 82 (1989) 171 [INSPIRE].

[50] Y. Katsuki et al., Z(4) and Z(6) orbifold models, Phys. Lett. B 218 (1989) 169 [InSPIRE].

[51] Y. Katsuki et al., Z(n) orbifold models, Nucl. Phys. B 341 (1990) 611 [InSPIRE].

[52] T. Kobayashi, H.P. Nilles, F. Ploger, S. Raby and M. Ratz, Stringy origin of non-abelian discrete flavor symmetries, Nucl. Phys. B 768 (2007) 135 [hep-ph/0611020] [INSPIRE].

[53] L.E. Ibáñez, R. Rabadán and A.M. Uranga, $\sigma$-model anomalies in compact $D=4, N=1$ type IIB orientifolds and Fayet-Iliopoulos terms, Nucl. Phys. B 576 (2000) 285 [hep-th/9905098] [INSPIRE].

[54] S.J.H. Konopka, Non Abelian orbifold compactifications of the heterotic string, JHEP 07 (2013) 023 [arXiv: 1210.5040] [INSPIRE].

[55] M. Fischer, M. Ratz, J. Torrado and P.K. Vaudrevange, Classification of symmetric toroidal orbifolds, JHEP 01 (2013) 084 [arXiv:1209.3906] [INSPIRE]. 
[56] M. Fischer, S. Ramos-Sanchez and P.K.S. Vaudrevange, Heterotic non-abelian orbifolds, JHEP 07 (2013) 080 [arXiv: 1304.7742] [INSPIRE].

[57] H.M. Lee et al., A unique $Z_{4}^{R}$ symmetry for the MSSM, Phys. Lett. B 694 (2011) 491 [arXiv: 1009.0905] [INSPIRE].

[58] H.M. Lee et al., Discrete $R$ symmetries for the MSSM and its singlet extensions, Nucl. Phys. B 850 (2011) 1 [arXiv:1102.3595] [INSPIRE]. 